\title{
An adaptive high-order hybrid scheme for compressive, viscous flows with detailed chemistry
}

\author{
Jack L. Ziegler \\ California Institute of Technology, 1200 E California Blvd., MC 205-45, Pasadena, CA 91125 \\ Ralf Deiterding
}

Oak Ridge National Laboratory, P.O. Box 2008 MS-6367, Oak Ridge, TN 37831

Joseph E. Shepherd, D. I. Pullin

California Institute of Technology, 1200 E California Blvd., MC 205-45, Pasadena, CA 91125

\begin{abstract}
A hybrid weighted essentially non-oscillatory (WENO)/centered-difference numerical method, with low numerical dissipation, high-order shock-capturing, and structured adaptive mesh refinement (SAMR), has been developed for the direct numerical simulation of the multicomponent, compressive, reactive Navier-Stokes equations. The method enables accurate resolution of diffusive processes within reaction zones. The approach combines time-split reactive source terms with a high-order, shock-capturing scheme specifically designed for diffusive flows. A description of the orderoptimized, symmetric, finite difference, flux-based, hybrid WENO/centered-difference scheme is given, along with its implementation in a high-order SAMR framework. The implementation of new techniques for discontinuity flagging, scheme-switching, and high-order prolongation and restriction is described. In particular, the refined methodology does not require upwinded WENO at grid refinement interfaces for stability, allowing high-order prolongation and thereby eliminating a significant source of numerical diffusion within the overall code performance. A series of oneand two-dimensional test problems is used to verify the implementation, specifically the high-order accuracy of the diffusion terms. One-dimensional benchmarks include a viscous shock wave and a laminar flame. In two space dimensions, a Lamb-Oseen vortex and an unstable diffusive detonation are considered, for which quantitative convergence is demonstrated. Further, a two-dimensional high-resolution simulation of a reactive Mach reflection phenomenon with diffusive multi-species mixing is presented.
\end{abstract}

Key words: weighted essentially non-oscillatory, detonation, adaptive mesh refinement, Navier-Stokes, direct numerical simulation, reacting compressible flow

\section{Introduction}

The compressible, reactive Navier-Stokes equations are a mixed-type set of partial differential equations (PDEs) with stiff source terms, presenting a significant challenge for numerical simulation. The result is a multiscale problem containing sharp gradients whose discretized solution is highly sensitive to numerical dissipation. The hyperbolic part of the equations (the inviscid terms as modeled by the Euler equations) is often solved numerically by using a shockcapturing method specifically designed to be stable when discontinuities are present. However, these robust methods introduce numerical dissipation that pollutes the diffusive part of the equations. For a convective-diffusive equation, owing to the physical viscosity, there are no discontinuities in a fully resolved solution, yet shock-capturing is still necessary to handle sharp gradients without spurious oscillations. Ideally, a method free of numerical dissipation is desired to capture accurately the diffusive terms. However, owing to their lack of shock-capturing dissipation, these schemes tend to lack robustness and stability in a structured adaptive mesh refinement (SAMR) framework. Because of these difficulties, a hybrid method has been developed, which combines the introduction of some numerical dissipation at sharp gradients, e.g., across shock waves, with a dissipation-free treatment with centered stencils in regions of smooth flow.

Email addresses: jackalak@caltech.edu(corresponding author) (Jack L. Ziegler), deiterdingr@ornl.gov (Ralf Deiterding), dale@galcit.caltech.edu (D. I. Pullin)

URL: www.galcit.caltech.edu/ jackalak (Jack L. Ziegler), http://www.csm.ornl.gov/ r2v (Ralf Deiterding), http://www.galcit.caltech.edu/EDL (Joseph E. Shepherd), http://fluids.caltech.edu (D. I. Pullin) 
The present numerical method combines and extends, both to high order and to the resolution of diffusive effects, numerical methods developed originally for large eddy simulation (LES) of turbulence [32, 56] and for efficient inviscid detonation simulation [19]. We employ a hybrid WENO/centered difference scheme [32, 56] with improved scheme switching [49]. Dynamic mesh adaptation is used to handle reactive flow problems of a multiscale nature, for which graded meshes (cf. Lele et al. [44]) are usually found insufficient. Our implementation is in flux form throughout, thereby ensuring conservation at scheme-matching boundaries, and uses a skew-symmetric form for the inertial fluxes [56], yielding improved overall kinetic energy conservation and stability. In general, our approach retains the benefits of low numerical dissipation away from shocks, the efficiency of an SAMR algorithm and the effectiveness of time-splitting for source term integration.

Aside from works such as [56, 50, 67, 53], which utilize the AMROC (Adaptive Mesh Refinement in Object-oriented $\mathrm{C}++)$ SAMR framework, hybrid WENO methods have mainly been applied on uniform or graded meshes. Recent examples include $[1,58,51,12,45,10]$. There has been much work in proving numerically and theoretically the stability of hybrid schemes. Adams et al. [1] addressed this problem and Larsson \& Gustafsson [45] give a detailed analysis, particularly proving the stability for a hybrid match between finite difference WENO and centered stencils. Their proof, using Kreiss or GKS theory [29], directly applies to our scheme's framework in the uniform case, yet, is difficult to extend to the SAMR case with overlapping grids. A brief discussion is provided in $\S 13.4$ of [29], where the stability of overlapping grids is theorized to be stable when viewed as an over-specified boundary value problem and deemed provable at least in the one-dimensional case.

Another high-order (in fact fourth-order accurate) method for use with SAMR has been developed for the Chombo SAMR software [11] based on a globally conservative finite volume formulation. This approach, while well adapted to inviscid simulations, can suffer from numerical dissipation in smooth flow regions, impeding its suitability for small-scale diffusive and viscous phenomena. The problem is shared by the classical second-order accurate MUSCL (Monotone Upstream-centered Schemes for Conservation Laws) methods and by most pure WENO schemes, thereby motivating the present work.

Diffusive effects in reacting flows have become a topic of recent research interest. Day et al. [13], using the BoxLib software, have reported lower-order accurate, SAMR-based, finite volume simulations for subsonic turbulent flames that model detailed chemistry and diffusive mixing. For their flows, the compressibility is small and this allows use of a low-Mach-number formulation. A hybrid essentially non-oscillatory (ENO)/centered-difference method with third-order Runge-Kutta (RK) time integration was used by Fedkiw [25] for the simulation of reacting flow. Uniform grids were employed. Related simulations of diffusive compressible reacting flow with detailed chemistry have been conducted by Massa et al. [52]. They utilized a third-order in time, fourth-order in space Rusanov, Kutler, Lomax, and Warming (RKLW) scheme as described by Kennedy \& Carpenter [40]. This method is not monotone or total variation diminishing (TVD) and a numerical filter, which is not appropriate for shock-capturing and SAMR, was used to damp spurious oscillations. Neglecting shock waves, a two-dimensional simulation of an equivalent shear layer behind triple points in detonations was conducted. Double Mach reflection (DMR) simulations with viscosity and thermal conduction have been reported by Vas Ilev et al. [36]. No-slip boundary conditions were used, which applies to shock-solid surface interactions rather than shock-shock interactions. Similar work has also been done for twoand three-dimensional, turbulent, compressible, reacting flow by Poludnenko et al. [59], using the fixed-grid massively parallel framework Athena-RFX. Their numerical method is based on a fully unsplit corner transport upwind (CTU) algorithm and an integration scheme using Colella-Woodward (PPM) spatial reconstruction in conjunction with an approximate nonlinear HLLC Riemann solver to achieve 3rd-order accuracy in space and 2nd-order accuracy in time.

The outline of this paper is as follows. In $\S 3$, WENO methods are briefly introduced and then extended to describe the symmetric variant and its hybridization with centered differences. Then in $\S 4$, the implementation of the scheme with time-split integration, discontinuity flagging, and flux-based SAMR is developed. One- and two-dimensional nonreactive and reactive verification problems are presented in $\S 5$. A brief introduction to compressible, reacting, diffusive flow is presented in $\S 6$. Finally, results are presented in $\S 7$ for a fully resolved reactive, diffusive DMR with one-step chemistry, along with preliminary unresolved results for multicomponent flow with detailed transport and chemistry. The present simulations can be viewed as extension and application of the hybrid approach to detonation-driven, diffusive-reactive flows within an SAMR framework, with a focus on the accurate resolution of reactive-diffusive effects.

\section{Reactive multicomponent Navier-Stokes equations}

To conduct direct numerical simulations (DNS) of detonation waves, the compressible Navier-Stokes equations are extended to model multidimensional, multicomponent, chemically reacting gas flows. The model assumes an ideal gas mixture with zero bulk viscosity. Soret and Dufour effects of mass diffusion, external body forces, and radiant heat transfer are neglected. This forms a large system of nonlinear conservation laws containing stiff source terms [25] and 
with both first- and second-order derivative terms from convective and diffusive transport. Mixture averaged transport is also assumed. In this approximation, "cross diffusion" terms are neglected and the solution of a matrix equation at each time step is avoided. Note that in this case, there are still separate temperature and pressure dependent diffusivities for each species. For the derivation, see [70].

\subsection{Formulation}

The problem is formulated for a mixture of $N$ species as

$$
\partial_{t} \mathbf{q}+\partial_{x} \mathbf{f}^{\text {conv }}+\partial_{y} \mathbf{h}^{\text {conv }}-\partial_{x} \mathbf{f}^{\text {diff }}-\partial_{y} \mathbf{h}^{\text {diff }}=\mathbf{s}^{\text {chem }}
$$

with vector of state $\mathbf{q}=\left(\rho u, \rho v, \rho e_{t}, \rho Y_{1}, \ldots, \rho Y_{N}\right)^{T}$ and the convective fluxes

$$
\mathbf{f}^{\text {conv }}=u \mathbf{q}+p(1,0, u, 0, \ldots, 0)^{T}, \quad \mathbf{h}^{\text {conv }}=v \mathbf{q}+p(0,1, v, 0, \ldots, 0)^{T},
$$

the diffusive fluxes

$$
\mathbf{f}^{\mathrm{diff}}=\left(\tau_{x x}, \tau_{x y}, u \tau_{x x}+v \tau_{x y}+k \partial_{x} T+\rho \sum_{i=1}^{N} h_{i} D_{i} \partial_{x} Y_{i}, \rho D_{1} \partial_{x} Y_{1}, \ldots, \rho D_{N} \partial_{x} Y_{N}\right)^{T}
$$

and

$$
\mathbf{h}^{\mathrm{diff}}=\left(\tau_{x y}, \tau_{y y}, u \tau_{x y}+v \tau_{y y}+k \partial_{y} T+\rho \sum_{i=1}^{N} h_{i} D_{i} \partial_{y} Y_{i}, \rho D_{1} \partial_{y} Y_{1}, \ldots, \rho D_{N} \partial_{y} Y_{N}\right)^{T}
$$

and the reactive source term

$$
\mathbf{s}^{\text {chem }}=\left(0,0,0,0, \dot{\omega}_{1}\left(T, p, Y_{1}, \ldots, Y_{N}\right), \ldots, \dot{\omega}_{N}\left(T, p, Y_{1}, \ldots, Y_{N}\right)\right)^{T},
$$

where $\tau$ denotes the stress tensor (defined in Appendix A). The mass fraction of the $i$-th species is computed from the partial and total density as $Y_{i}=\rho_{i} / \rho$. The enthalpy of the gas mixture is denoted by $h ; \dot{\omega}_{i}$ is the mass production rate of the $i$-th species, $\mu$ the mixture viscosity, $k$ the mixture thermal conductivity, and $D_{i}$ the mass diffusivity of the $i$-th species into the mixture. The mass production rates are specified by Arrhenius rate equations, determined by the particular reaction mechanism. The contribution of each species to the total energy is obtained by using a mass-fraction-averaged enthalpy, i.e.,

$$
h=\sum_{i=1}^{N} Y_{i} h_{i}, \quad \text { where } \quad h=e_{t}+\frac{p}{\rho}-\frac{u^{2}+v^{2}}{2} .
$$

To close the system of equations, we have the ideal gas law for the average mixture properties, as derived from the partial pressure equation for each species, that reads

$$
p=\sum_{i=1}^{N} p_{i}=\sum_{i=1}^{N} \rho Y_{i} R_{i} T=\rho R T \quad \text { with } \quad R=\sum_{i=1}^{N} Y_{i} R_{i}, \quad R_{i}=\frac{\mathcal{R}}{W_{i}},
$$

where $\mathcal{R}$ is the universal gas constant and $W_{i}$ the molar mass of each species. The mass production rates are computed by summing the contributions from each chemical reaction. The reactions are all formulated with an Arrhenius rate equation, cf. [70]. The sound speed, $a$, a parameter that is calculated throughout the simulation, must be derived for a mixture, of thermally perfect, ideal gases. The frozen sound speed is defined as the derivative of the pressure with respect to the density at constant entropy and species concentrations and reads

$$
a^{2}=\left(\frac{\partial p}{\partial \rho}\right)_{s, Y_{1}, \ldots, Y_{N}}=\gamma R T=\gamma \frac{p}{\rho}=\gamma \sum_{i=1}^{N} Y_{i} T
$$

Using a representation of each species' specific heat at constant pressure and constant volume, denoted by $c_{p i}(T)$ and $c_{v i}(T)$, respectively, as a function of $T$, the specific heat ratio for each species is

$$
\gamma_{i}(T)=\frac{c_{p i}(T)}{c_{v i}(T)}, \quad c_{v i}=c_{p i}(T)-R_{i} .
$$

A mixture value for $\gamma-1$ is found using the mole fraction, $X_{i}$, as

$$
\gamma(T)=1+\left(\sum_{i=1}^{N} \frac{X_{i}}{\gamma_{i}(T)-1}\right)^{-1}, X_{i}=\frac{\rho_{i}}{\rho}=\frac{Y_{i} W}{W_{i}} \quad \text { with } \quad W=\left(\sum_{i=1}^{N} \frac{Y_{i}}{W_{i}}\right)^{-1}=\sum_{i=1}^{N} X_{i} W_{i} .
$$

For the canonical eigendecomposition, for instance, of the Jacobian of the flux function $\mathbf{f}^{\text {conv }}$, see Appendix C. 


\subsection{Temperature evaluation}

Thermally perfect gases require the computation of the temperature from the conserved quantities by solving an implicit equation. Consider the pressure as the sum of partial pressures,

$$
p=\sum_{i=1}^{N} \rho Y_{i} \frac{\mathcal{R}}{W_{i}} T
$$

and also as related to the enthalpy and internal energy of the mixture,

$$
p=H-E=\sum_{i=1}^{N} \rho_{i} h_{i}(T)+\rho e_{t}, \quad p=\sum_{i=1}^{N} \rho_{i} h_{i}(T)-\rho e_{t}+\rho \frac{u^{2}+v^{2}}{2} .
$$

The pressure in (11) and (12) must be equal, giving the equation

$$
0=\sum_{i=1}^{N} \rho_{i} h_{i}(T)-\rho e_{t}+\rho \frac{u^{2}+v^{2}}{2}-\sum_{i=1}^{N} \rho Y_{i} \frac{\mathcal{R}}{W_{i}} T
$$

\section{Numerical method}

The shock-capturing component of the present hybrid schemed uses a sixth-order accurate, symmetric enhancement of the classical WENO scheme as developed by Shu [37, 65]. In the limit of smooth flow, the method obtains a 6thorder centered stencil. We presently utilize only hybrid WENO/centered-difference schemes for SAMR. Alternatives could also be pure WENO schemes with enhanced smoothness measures, such as the Mapped WENO schemes [30, 8], or schemes with improved stability properties, such as the monotonicity preserving schemes [4]. Also of interest for non-steady, reactive, compressible flows is the implementation of WENO with one-dimensional shock-fitting [31].

In the following section, WENO schemes are briefly described and the extension to a symmetric WENO method is elaborated. Also, the time-split approach for reactive source terms, when using a Runge-Kutta integrator, is detailed.

\subsection{ENO and WENO schemes for conservation laws}

ENO and WENO discretizations [65] have revolutionized the solution of nonlinear hyperbolic conservation laws, particularly for the multidimensional Euler equations, which are often used as an inviscid, hyperbolic, non-diffusive approximation of the mixed-type Navier-Stokes equations. This approximation is suited for the simulation of shock waves, contact discontinuities, and other non-smooth flow features. ENO and WENO schemes have been specifically developed for problems containing both piecewise smooth solutions and discontinuities. They are designed to obtain arbitrarily high order of accuracy in smooth solution regions while minimizing the propagation of first-order errors obtained along discontinuities. WENO schemes are an extension of ENO. Instead of choosing from a group of stencils, the WENO approach uses a convex combination of all stencils. Smoother stencils are given larger weight, yet, at discontinuities, WENO performs exactly as ENO. In the limit of smooth regions, however, the WENO approach obtains a much higher order of accuracy. ENO and WENO schemes can either be finite difference or finite volume, yet, the finite difference variant is commonly chosen for reasons of efficiency [65]. For the finite difference schemes, the wider the interpolation stencil, the higher the order of accuracy obtained; however, this is only true provided the solution is smooth. Using centered stencils at discontinuities causes undesirable oscillations in the numerical solution. These oscillations propagate through the domain and can create instabilities in the numerical solution.

When discontinuities are present, the global order of accuracy is always reduced to one [28]. ENO, WENO, and, for example, discontinuous Galerkin finite volume methods will not by themselves obtain high order of accuracy near discontinuities. Therefore, these schemes are often referred to as high-resolution rather than high-order methods. In fluid mechanics, whenever shocks are captured (rather than resolved as possible with the Navier-Stokes equations), first-order accuracy is obtained. Note that high-resolution schemes are nonetheless useful because complex structures, not resolved by low-order methods, can be studied in greater detail. High order of accuracy can be achieved in some cases, for instance, when a shock-fitting method is used [31]. Here, the discontinuity's velocity and shape is tracked, and at each time step the Rankine-Hugoniot equations for the shock are solved exactly. This is easily implemented for one-dimensional problems, yet too expensive in two and three space dimensions. Difficulties also arise on how to track complex multidimensional structures, cf. [14]. Thus, for all but the simplest problems, shock-capturing methods are currently still needed. 


\subsubsection{WENO reconstruction}

For each interpolated flux, the smoothest stencil is chosen among the group of neighboring stencils. In a $k$-th-order ENO scheme, $k$ candidate stencils are considered over a range of $2 k-1$ cells, but only one stencil is used in the reconstruction. The basis of WENO is to take advantage of this by using a convex combination of all of them. Two properties are desired: ENO-like performance at discontinuities and the usage of all $k$ stencils in the limit of a smoothly varying solution, yielding $O\left(\Delta x^{2 k-1}\right)$ convergence. The convex combination is a linear combination of fluxes, where all coefficients (smoothness-biased weights), $\omega_{r}$, are non-negative and sum up to one, i.e., $\omega_{r} \geq 0$ for $r=0, \ldots, k-1$ and $\sum_{r=0}^{k-1} \omega_{r}=1$. The results from each stencil, $S_{r}=\left\{x_{j-r}, \ldots, x_{j-r+k-1}\right\}$, are combined with the weights $\omega_{r}$ to obtain the approximation of the boundary fluxes $\hat{f}$. The combination yields

$$
\hat{f}_{j+1 / 2}=\sum_{r=0}^{k-1} \omega_{r} c_{r \kappa} f_{j-r+\kappa} .
$$

Since we are using a structured non-graded mesh, the same set of coefficients, $c_{r \kappa}$, is used for each point value. This leads to an approximation of the derivative at the cell center in terms of point fluxes at the cell boundaries. The weights are designed for the limit of smoothly varying flow. In this limit, all smoothness measures are equal and the weights become the predetermined values such that all $k$ stencils together, act as one large stencil that interpolates the $2 k-1$ cells, hence obtaining $(2 k-1)$-th order of accuracy. The weights $\omega_{r}$ approach the coefficients $d_{r}$, which, when multiplied appropriately by each stencil's coefficients $c_{r \kappa}$ and summed, become exactly those coefficients for a polynomial with $2 k-1$ interpolation points. Hence, in the limit of smooth solutions, the weights are equal to $d_{r}$, while for a discontinuity, some or all weights become zero, depending on if the discontinuity is located within or at the boundary of a cell. With a smoothness measure $\beta_{r}$ for the $r$-th stencil defined, the weights are

$$
\omega_{r}=\frac{\alpha_{r}}{\sum_{s=0}^{k-1} \alpha_{s}} \quad \text { with } \quad \alpha_{r}=\frac{d_{r}}{\left(\epsilon+\beta_{r}\right)^{2}},
$$

where $\epsilon>0$ is an arbitrary problem- and mesh-size-dependent parameter which can range from $10^{-2}$ to $10^{-30}$. Shu [65] recommends $10^{-6}$. Depending on the type of derivative approximation (for example, with centered differences), numerous formulas for $\beta_{r}$ are possible. Thus, measures reported in the literature are varied. Note that in deriving a smoothness measure, it is still required that the $\beta_{r}$ become zero when a constant solution is encountered in order to ensure convergence to $(2 k-1)$-th order in smooth solution regions.

The optimal $(2 k-1)$-th-order stencil can be biased in a particular direction for upwinding or is unbiased for a symmetric centered scheme. For the present DNS, an optimal, symmetric, centered stencil is desired, because the numerical dissipation created otherwise pollutes the sensitive nature of the diffusive part of the solution.

\subsubsection{Characteristic form}

For the finite difference scheme, aside from solving the exact Riemann problem at the cell boundaries, the highest accuracy is obtained if a characteristic decomposition is adopted. Here, the system of equations are diagonalized using a local approximation of the Jacobian at each cell boundary. The state $\mathbf{q}_{j}$ and flux $\mathbf{f}\left(\mathbf{q}_{j}\right)$ are transformed into the characteristic state $\mathbf{v}_{j}$ and flux $\mathbf{g}\left(\mathbf{q}_{j}\right)$, as detailed by Shu [65]. The exact transformation is determined by the local eigenvectors at the cell boundary. It is unknown and must be approximated. The simplest way of doing this is to use the average state at the right and left cells. For fluid mechanics, an expensive but more accurate method is to use the Roe average, which is suitable for shock waves,

$$
\mathbf{f}_{j+1}-\mathbf{f}_{j}=\mathbf{f}_{j+\frac{1}{2}}^{\prime}\left(\mathbf{q}_{j+1}+\mathbf{q}_{j}\right)\left(\mathbf{q}_{j+1}-\mathbf{q}_{j}\right),
$$

from which the eigenvectors of the Jacobian, $\mathbf{f}_{j+\frac{1}{2}}^{\prime}$, can be determined. Note that the canonical eigendecomposition of the inertial fluxes of the Navier-Stokes equations is provided for completeness in Appendix C. One important step remains before transforming the fluxes back from the characteristic space. A flux split is conducted, which separates the left- and right-moving contributions, based on the negative and positive characteristics. The most commonly used flux split is the Lax-Friedrichs flux splitting, where for the $m$-th component of the flux

$$
g_{m}^{ \pm}(\mathbf{v})=\frac{1}{2}\left(g_{m}(\mathbf{v}) \pm \alpha_{m} v_{m}\right)
$$

is used. The coefficient $\alpha_{m}$ is taken as the maximum of the range of the $m$-th eigenvalue in the solution over the whole domain, i.e.,

$$
\alpha_{m}=\max _{j}\left|\lambda_{m, j}\left(\mathbf{q}_{j}\right)\right|
$$




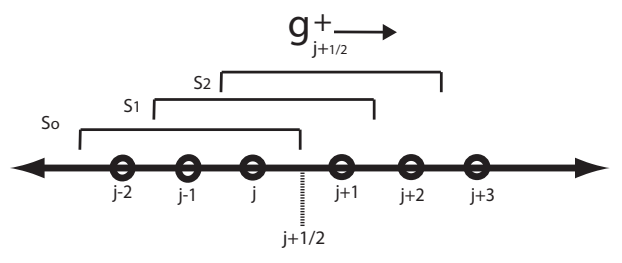

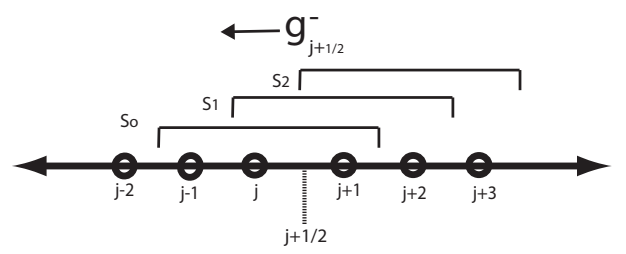

(a) 5th-order Shu stencil

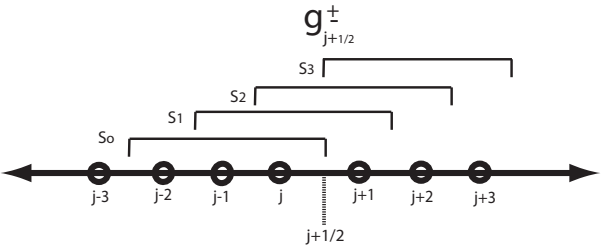

(b) 6th-order Weirs stencil

Figure 1: Set of candidate stencils for two differente finite difference WENO methods using flux splitting. The positive and negative characteristic fluxes, $g^{+}$and $g^{-}$, respectively, are calculated at the cell boundary located at $x_{j+\frac{1}{2}}$.

With the positive and negative characteristic fluxes found, the finite-difference reconstruction procedure is used twice to derive the positive and negative fluxes at the cell boundaries. As shown in Figure 1(a), two different sets of stencils and optimal stencils are used. In two space dimensions, the final form of the approximation of the point-wise time derivative for the $(j, l)$-th cell is

$$
\frac{d \mathbf{q}_{j l}}{d t}=-\frac{1}{\Delta x}\left(\hat{\mathbf{f}}_{j+\frac{1}{2}, l}-\hat{\mathbf{f}}_{j-\frac{1}{2}, l}\right)-\frac{1}{\Delta y}\left(\hat{\mathbf{h}}_{j, l+\frac{1}{2}}-\hat{\mathbf{h}}_{j, l-\frac{1}{2}}\right) .
$$

\subsection{Enhanced WENO schemes}

WENO schemes perform well for purely hyperbolic PDEs, yet, for mixed equations with physical diffusion, they introduce too much numerical dissipation that tends to artificially remove energy from the highest resolved wave numbers [68]. This numerical damping arises from the upwinded, optimal stencils and the smoothness measures. Traditionally, finite difference approximations have been designed to maximize the order of accuracy. However, contemporary research interest in turbulent flow has expressed the need for minimizing the approximation error of the small turbulent scales. The resulting finite difference schemes are based on bandwidth-optimized stencils (see [46]). For our application, DNS, these schemes are the basis for a more general order-optimized variant. Our order-optimized scheme includes the symmetric WENO stencils first developed for bandwidth-optimized schemes, e.g., WENO-SYM [68]. Our implementation, which we call WENO/CD, is a hybrid approach, extended to an SAMR framework and configured for order optimization.

\subsubsection{WENO-SYM}

Weirs [68] used bandwidth optimization techniques to develop symmetric optimal stencils with reduced dissipation and greater resolving efficiency. Using Fourier analysis, the coefficients are chosen to resolve the high frequencies of interest instead of tailoring them for maximal order of accuracy. In this particular case, the optimal 5th-order 5-point stencil, originally constructed with three 3-point stencils, is changed to a symmetric 6th-order, 6-point stencil that is constructed with four 3-point stencils, as shown in Figure 1(b). Then by bandwidth (rather than order) optimizing the coefficients, a 4th-order accurate optimal stencil is found with the desired spectral properties.

Note the differences between the WENO-SYM stencil and that of the original WENO as depicted in Figure 1(a). The optimal WENO-SYM stencil is centered at the point at which the flux is being evaluated while the optimal WENO stencil is upwinded in order to mimic the flux in the characteristic directions. The latter is advantageous for correctly modeling the flow of information but introduces numerical dissipation which is undesirable for convergence when resolving small turbulent scales and diffusive mixing. In contrast to Shu's upwinded counterparts [65] only the smoothness measurement introduces dissipation into the WENO-SYM approach, cf. [68]. Now with an extra stencil present and all stencils shifted as shown in Figure 1(b), the WENO-SYM construction is performed with the unchanged stencils $S_{r}$ but uses $\omega_{r} \geq 0$ for $r=0, \ldots, k$ with $\sum_{r=0}^{k} \omega_{r}=1$ and thereby reads

$$
\hat{f}_{j+1 / 2}=\sum_{r=0}^{k} \omega_{r} c_{r \kappa} f_{j-r+\kappa} .
$$


In the limit of smooth flow all $k+1$ stencils together act as one large stencil, which interpolates $2 k$ cells obtaining $(2 k)$-th order of accuracy. For the formally 6th-order accurate WENO-SYM scheme, four 3rd-order accurate ENO stencils are used. In this order-optimized implementation for $k=3$, the optimal weights, $d_{r}$, and the ENO stencils, as specified by $c_{r \kappa}$, are

$$
d_{r}=\left\{\frac{1}{20}, \frac{9}{20}, \frac{9}{20}, \frac{1}{20}\right\}, c_{1, \kappa}=\left\{\frac{2}{6}, \frac{-7}{6}, \frac{11}{6}\right\}, c_{2, \kappa}=\left\{\frac{-1}{6}, \frac{5}{6}, \frac{2}{6}\right\}, c_{3, \kappa}=\left\{\frac{2}{6}, \frac{5}{6}, \frac{-1}{6}\right\}, c_{4, \kappa}=\left\{\frac{11}{6}, \frac{-7}{6}, \frac{2}{6}\right\} .
$$

The evaluation of the smoothness measure for the WENO-SYM scheme is complicated by the fact that a discontinuity could be located exactly at the center of the stencil. This problem is effectively avoided by forcing the right most stencil to have a smoothness parameter, $\beta_{k}$, equal to zero when calculating the positive characteristic flux, $\mathbf{g}_{j+\frac{1}{2}}^{+}$, and similarly forcing the left most stencil to have $\beta_{0}$, equal to zero for the negative characteristic flux, $\mathbf{g}_{j+\frac{1}{2}}^{-}$. For the positive characteristic flux with $k=3$ and $\beta_{3}=0$, the remaining smoothness measures for $r=0, \ldots, 2$ are defined as

$$
\beta_{r}=\sum_{n=1}^{2}\left(\sum_{l=1}^{3} d_{r, n, l} f\left(q_{j-k+1+r+l}\right)\right)^{2}
$$

where the smoothness coefficients, $d_{r, n, l}$, are given by

$$
d_{1,1, l}=\left\{\frac{1}{2}, \frac{-4}{2}, \frac{3}{2}\right\}, \quad d_{2,1, l}=\left\{\frac{-1}{2}, 0, \frac{1}{2}\right\}, \quad d_{3,1, l}=\left\{\frac{3}{2}, \frac{-4}{2}, \frac{1}{2}\right\}, \quad d_{4,1, l}=\left\{\frac{-5}{2}, \frac{8}{2}, \frac{-3}{2}\right\},
$$

and

$$
d_{r, 2, l}=\left\{\sqrt{\frac{13}{12}},-2 \sqrt{\frac{13}{12}}, \sqrt{\frac{13}{12}}\right\}, \quad r=0, \ldots, 3 .
$$

In the limit of smooth flow, all smoothness measures approach zero. Thus, for example, when WENO-SYM is used near but not at shock waves, the equivalent WENO interpolation is still approaching a perfect, zero-dissipation, centered stencil.

\subsubsection{WENO/CD}

Hill et al. [32] and Pantano et al. [56] developed a robust hybrid WENO/tuned centered difference (TCD) method, which combines the TCD stencil with the WENO-SYM scheme. The centered difference stencil was bandwidthoptimized, specifically for weakly compressible decaying turbulence [56]. The optimal WENO weights are chosen to match those of the TCD scheme thereby minimizing oscillations at the matching boundaries. The location of the scheme-switching boundary is defined by a problem dependent switch. By using the relatively inexpensive TCD stencil predominantly in regions where the solution is smooth and WENO-SYM at and around discontinuities, the overall resulting WENO/TCD scheme performs faster and additionally has the spectral resolution desired in turbulence simulations. For DNS, however, where all scales are resolved, a symmetric order-optimized stencil is ideal. Therefore, for our application a WENO/CD rather than WENO/TCD method is used.

For schemes based on centered stencils, no numerical viscosity is present, yet care is needed to avoid nonlinear instabilities that may develop [56]. Such instabilities can be alleviated by using a skew-symmetric formulation that conserves the kinetic energy [34] and prevents the convective terms of the momentum and energy equations from artificially producing or dissipating global kinetic energy. Without this, it has been found that in unstable flow simulations, the entropy of the system decreases with time, a clear violation of the second law of thermodynamics.

\subsection{Stability}

For WENO and other high-order spatial methods, at least second-order TVD (Total variation Diminishing) time integration is required for stability. Higher order integrators, often up to fifth order, have been used, but can be computationally very expensive. Also, in viscous and reactive-flow simulations the approximation error due to the spatial discretization is generally dominant. Thus a higher-order spatial, rather than temporal discretization is used. Additionally, any temporal discretization exhibits its own numerical dissipation, but whose influence is typically much smaller than that introduced by the WENO interpolation or the upwinding. Recent research on the time integration of compressible flows has generally focused on efficiency and stability rather than the reduction of temporal numerical dissipation.

The commonly chosen time discretization for hyperbolic problems is the method of lines. The most popular methods used with the Euler equations are explicit TVD or strong stability preserving Runge-Kutta (SSPRK) methods, where each of these is designed for a specific $n$-th order of time accuracy, $O\left(\Delta t^{n}\right)$. Also, note that each TVD scheme has 
a critical Courant-Friedrichs-Lewy (CFL) number, $\nu$, above which the method is not guaranteed to be stable. A typical definition (for the 1D inviscid case only) would be $\nu:=\frac{\Delta t}{\Delta x}\left|\lambda_{\max }\right| \leq 1$, where $\lambda_{\max }$ is the maximal eigenvalue encountered in the domain at a particular time, $t,[47]$. For PDEs with diffusion and convection, the latter relation needs to adjusted (see Appendix B for the case of two-dimensional reactive Navier-Stokes equations).

Note that in practice we use the stability limits of linear systems of equations for our nonlinear system. The stability of a numerical method can be discussed in terms of having a bounded norm. For smooth problems, linearizing the equations and using, for instance, the $L_{2}$-norm is sufficient. However, for problems with discontinuities, a bounded $L_{2}$-norm does not ensure that the solution is non-oscillatory. For a non-oscillatory solution, at least the TV (total variation) or similarly the $L_{\max }$-norm must exist [27]. The TV semi-norm is defined as $\|u\|_{T V}=\sum_{j=0}^{N}\left|u_{j+1}-u_{j}\right|$.

Finite volume methods with order of accuracy $>2$ have not been proven to be TVD [54]. The intrinsic complexity of WENO and hybrid methods with order $\geq 3$ has made any analytical proof of non-oscillatory solutions difficult. In practice, SSPRK integration is therefore used for WENO methods. The SSP property is a very strong requirement that guarantees strong monotonicity in arbitrary convex functionals, for arbitrary starting values and arbitrary nonlinear, nonautonomous equations [27]. The stability in the TVD sense of these methods is ensured for a particular CFL parameter, $\nu$, if the spatial discretization is also stable when used with the 1st-order accurate forward Euler method for temporal integration. However, proof of the TVD stability of WENO methods, that use spatial reconstruction with order 3 or higher, cannot be made as any such operator is linear unstable when combined with the forward Euler method [66] or any explicit Runge-Kutta method with less than third-order accuracy. Nevertheless, SSPRK methods work well in practice with WENO operators and provide essentially non-oscillatory solutions.

Therefore, to date, in order to make some estimates of the stability region, analogies with 2nd-order accurate spatial discretizations with forward Euler time-integration are commonly used. Note that the MUSCL method is 2nd-order accurate both in space and time, and hence, its stability limit of $\nu \leq 1$ cannot be used as an analogy (cf. [47]). WENO/ENO methods with second-order spatial accuracy are equivalent to slope-limited MUSCL schemes. When combining these methods with the forward Euler method in time, the analytical nonlinear TVD stability result (when using example equations such as the advection equation and Burger's equation and Harten's lemma) ranges from $\nu \leq \frac{1}{2}$ to $\nu \leq \frac{2}{3}$ [27], depending on the limiter. It is surmised that WENO/ENO hybrid methods should perform similarly; yet, for numerous problems, such as the ones presented in here, they perform so well in practice that convergence in the $L_{1}$ - and $L_{2}$-norm can be established for $\nu \leq 1$ (see below). This by no means proves that using $\nu \leq 1$ will work for all possible solutions of the Euler and Navier-Stokes equations; a smaller value, such as $\nu \leq \frac{1}{2}$, is a generally safe choice. Note, that for a full study of stability properties, an analysis of the convergence in the $L_{\max }$-norm is also needed.

\subsection{Time discretization}

The typical third-order, three-step $\operatorname{SSPRK}(3,3)$ scheme, integrating from time step $n$ to $n+1$, is

$$
q^{(1)}=q(n)+\Delta t L(q(n)), \quad q^{(2)}=\frac{3}{4} q(n)+\frac{1}{4} q^{(1)}+\frac{1}{4} \Delta t L\left(q^{(1)}\right), \quad q(n+1)=\frac{1}{3} q(n)+\frac{2}{3} q^{(2)}+\frac{2}{3} \Delta t L\left(q^{(2)}\right) .
$$

In this case, the linear stability limit is $\nu \leq 1.434$ [27]. For our nonlinear problem we used the limit $\nu \leq 1$. This method has an SSP coefficient $c=1$, thereby permitting a time step size similar to the forward Euler method. The costs of the overall method are three times a forward-Euler update. In the last equation, $L(q)$ is the numerical approximation of the spatial differential operator of the hyperbolic equation. For smooth solutions approximated with a three-step Runge-Kutta method (RK3) and sixth-order accurate WENO, we therefore have

$$
q_{t}=L(q)+O\left(\Delta x^{6}\right)+O\left(\Delta t^{3}\right) .
$$

When viscous scales are also being resolved, we must consider how numerical viscosity from the spatial discretization scales with the CFL number. Using the advection-diffusion equation as an example, if one uses first-order Euler time integration, upwinding on the advection term and a centered difference approximation of the diffusive term, one finds that the numerical viscosity from the space discretization (which scales with $\Delta x^{4}$ in this case) approaches zero as the CFL number approaches one. Therefore, for mixed-type PDEs with advection and diffusion terms, the highest possible stable time step should be used.

For the reactive simulations, owing to the large difference in time scales between the fluid dynamics and the reactive source terms, a time-splitting method is used in combination with the $\operatorname{SSPRK}(3,3)$ method of Ketchenson et al. [27]. The stiff source terms are integrated separately in each cell utilizing the 4th-order accurate semi-implicit GRK4A method of Kaps and Rentrop [38], which avoids a globally coupled implicit problem. Using the Strang splitting approach, the maximal temporal order of accuracy is limited to two [47]. An easily neglected detail for Runge-Kutta schemes is the proper time update within the sub-steps, which is vital to ensure the correctness of time-dependent boundary conditions, as used, for instance, at SAMR-level boundaries when hierarchical time step refinement is employed. Therefore, we sketch the application of Strang splitting with $\operatorname{SSPRK}(3,3)$ below. With $s_{\text {step }_{\iota}}=\left(1,-\frac{1}{2}, \frac{1}{2}\right)$ the algorithm reads: 


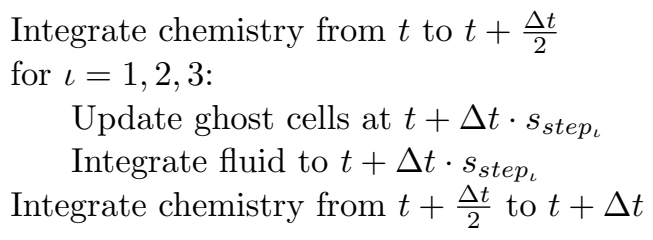

Also, for the non-reactive preliminary simulations, a 10-step, 4th-order Runge-Kutta scheme, $\operatorname{SSPRK}(10,4)$ [27], is used. While typically higher than 3rd-order SSP/TVD Runge-Kutta schemes have a stability coefficient smaller than one, for this unique scheme the stable region is for $\nu=6$. This scheme is more efficient than $\operatorname{SSPRK}(3,3)$, however, is more difficult to integrate with a time-splitting scheme owing to its 10 as compared to 3 sub-steps. The 4 th-order scheme is stable for the collective 10 steps with a CFL number six times larger than that of the 3rd-order scheme with its collective 3 steps, leading to a scheme that is almost twice as efficient. This 4th-order scheme can also be compared to the four-step 4th-order scheme, $\operatorname{SSPRK}(4,4)$, which has $\nu=0.624[26]$. Note that $\nu$ corresponds to the total time-step as computed from the contribution of all sub-steps. The $\operatorname{SSPRK}(10,4)$ scheme reads:

$$
\begin{aligned}
& \text { for } \iota=1, \ldots, 10 \text { : } \\
& \text { if }(\iota \neq 10): \quad q^{(\iota)}=q^{(\iota-1)}+\frac{\Delta t}{6} L\left(q^{(\iota-1)}\right) \\
& \text { if }(\iota=5): \quad q^{(5)}=q^{(4)}+\frac{\Delta t}{6} L\left(q^{(4)}\right), \quad q^{(*)}=\frac{1}{25} q^{n}+\frac{9}{25} q^{(5)}, \quad q^{(5)}=15 q^{(*)}-5 q^{(5)} \\
& \text { if }(\iota=10): \quad q(n+1)=\frac{6}{10} q^{(9)}+\frac{\Delta t}{10} L\left(q^{(9)}\right)+q^{(*)}
\end{aligned}
$$

\section{SAMR implementation}

We utilize the fluid-solver framework AMROC, version 2.0, integrated into the Virtual Test Facility [21, 22, 23], which is based on the block-structured adaptive mesh refinement algorithm pioneered by Berger \& Oliger [7] and refined by Berger \& Colella [6]. This algorithm is designed especially for the solution of hyperbolic partial differential equations with SAMR, where the computational grid is implemented as a collection of rectangular grid components. Finite-difference methods are limited to either uniform grids or SAMR, while the finite-volume approach can also be used with unstructured meshes. The SAMR method follows a patch-wise refinement strategy. Cells are flagged by error indicators and clustered into rectangular boxes of appropriate size. Refined grids are derived recursively from the coarser level and a hierarchy of successively embedded grid patches is constructed. With its parallel distribution strategy, described in detail in [15] and [19], AMROC synchronizes the overlapping ghost cell regions of neighboring patches user-transparently over processor borders whenever boundary conditions are applied. An efficient partitioning strategy for distributed memory machines is used for high-performance simulations with MPI-library, cf. [15].

Typically, a second-order accurate Cartesian finite volume method (commonly of the MUSCL type) is used with SAMR implementations. AMROC has been employed very successively with such schemes to efficiently simulate shockinduced combustion, particularly detonation waves, with simplified [9] and detailed chemical kinetics [16, 18]. From the standpoint of DNS, however, the low numerical dissipation of the 6th-order hybrid WENO/CD scheme should provide faster grid-wise convergence than a second-order scheme. Also, it is predicted that for three-dimensional simulations, owing to the multiscale nature of the problem, it will be very expensive to obtain the desired resolution needed in the diffusive mixing and reaction zones. In the case where the simulation is memory rather than compute time limited (i.e., the available memory determines the highest possible resolution), the 6th-order accurate method yields superior results.

In order to prescribe non-rectangular domains in $\S 7$ we utilize a level-set-based embedded boundary method, that has been demonstrated and verified in detail for chemically reactive flows in [17].

\subsection{Centered differences in flux-based form}

WENO schemes themselves are naturally flux-based formulations, but a flux-based formulation of the centereddifference method is also required in order to enforce conservation at the WENO-SYM/CD scheme-matching points. For the $j$-th point at the cell center, the flux in the $x$-direction can be approximated with a 7 -point centered stencil as

$$
\left.\frac{\partial f}{\partial x}\right|_{j} \approx \frac{1}{\Delta x}\left(\alpha\left(f_{j+3}-f_{j-3}\right)+\beta\left(f_{j+2}-f_{j-2}\right)+\gamma\left(f_{j+1}-f_{j-1}\right)\right),
$$

where $\alpha=1 / 60, \beta=-3 / 20$, and $\gamma=3 / 4$ are constants selected for the 6 th-order accurate stencil. However, in order to enforce conservation, we must consider the fluxes at the cell boundaries $f_{j+\frac{1}{2}}$ and $f_{j-\frac{1}{2}}$. For the inertial fluxes, the local $f_{j}$ at the cell centers are explicitly known. However, the local cell-centered diffusive fluxes must be calculated 
with a stencil similar to that of (27) as discussed in $\S 4.2$. These together are used to obtain the fluxes at the cell walls, obtaining the final desired conservative approximation of the flux at the cell center, i.e.,

$$
\left.\frac{\partial f}{\partial x}\right|_{j} \approx \frac{1}{\Delta x}\left(f_{j+\frac{1}{2}}-f_{j-\frac{1}{2}}\right)
$$

where $f_{j+\frac{1}{2}}$ and $f_{j-\frac{1}{2}}$ are calculated using

$$
f_{j+\frac{1}{2}}=\left(\bar{\alpha}\left(f_{j+3}+f_{j-2}\right)+\bar{\beta}\left(f_{j+2}+f_{j-1}\right)+\bar{\gamma}\left(f_{j}+f_{j+1}\right)\right)
$$

with $\bar{\alpha}=1 / 60, \bar{\beta}=-2 / 15$, and $\bar{\gamma}=37 / 60$. However, directly using this is not advised. Presently, we utilize the skewsymmetric form on each piece of the decomposed flux (see Pantano et al. [56] for details). The derivative of the flux at the cell center is obtained by adding the contributions from the cell boundaries in (28). Note that in this formulation, conservation is ensured but at the cost of a second-order error at cell interfaces where the centered difference (CD) stencil is matched with the WENO-SYM scheme. Since WENO is only used at shock waves, this loss of accuracy is negligible compared to the dominating first-order error along the discontinuity. The forced conservation ensures the stability of the method in an SAMR framework.

\subsection{Diffusive-flux approximation}

The approximation of the diffusive fluxes shares the previously encountered problem that the numerical fluxes must be calculated at the cell walls in a conservative fashion. For orders of accuracy greater than two, it is difficult to obtain derivatives (nonlinear combinations of first and second derivatives) at the cell walls without allowing for stencil widening. To obtain a 6th-order accurate diffusive-flux approximation, the fluxes need to be first evaluated at the cell centers. However, in order to obtain these approximations, first derivatives of the velocities, mass fractions, and temperatures, must be calculated. For this a standard 7-point centered stencil for the derivative is used. As an example for the velocity, the derivative in the $y$-direction of the $v$-velocity is calculated as

$$
\left.\frac{\partial v}{\partial y}\right|_{l}=\frac{1}{\Delta y}\left(\alpha\left(v_{l+3}-v_{l-3}\right)+\beta\left(v_{l+2}-v_{l-2}\right)+\gamma\left(v_{l+1}-v_{l-1}\right)\right),
$$

where $l$ denotes the cell index in the $y$-direction. The appropriate diffusive-flux terms are then known at the cell centers with 6th-order accuracy. Yet, for the SAMR framework, fluxes at the cell walls are needed. Therefore, these cell-centered values are interpolated to the cell walls using (28) such that, when the wall fluxes are combined with (29), the resulting flux at the cell center is conservative at the scheme boundaries and of the desired 6th order of accuracy. In this case, the final cell-centered flux is influenced by a total of 12 cells for each direction. Hence, 6 ghost/boundary cells are required in the SAMR implementation. One may devise a way to calculate the wall fluxes directly in a way that only uses 6 cells for 6th-order accuracy, however, difficulties arise when attempting to calculate cross-derivative terms, such as $\tau_{x y}$ (cf. Appendix A), as it is not straightforward to simultaneously use less than 12 cells and ensure a conservative form.

\subsection{Hybrid method boundary flagging}

The WENO/CD scheme requires an explicit switch, from regions of smooth flow (using CD) to regions of extremely high gradients, that require WENO, such as shocks. The normalized curvature of pressure and density was used by Hill \& Pullin [32]. However, this flagging criterion can be sensitive to the resolution of the mesh in the sense that a smooth flow can appear almost discontinuous if a sufficiently coarse mesh is employed. The problems encountered with this switch motivated development of an alternative, shock-detection based technique [49]. The new technique is robust, precise and efficient. While reminiscent of shock-fitting in terms of location strong shock waves, it preserves efficiency by not requiring a deformable mesh.

The shock-based detection is suitable for the high-speed DMR problems, studied in depth below, because the shocks continually bound the mixing layer as shown in Figure 2. The technique uses an approximate Riemann solver to detect the existence and orientation of strong shock waves, while ignoring weak ones. The approximate solution to the Riemann problem is computed using Roe-averaged quantities from the given left $(L)$ and right $(R)$ state. Liu's entropy condition allows for characterizing the type of the wave encountered at the characteristic associated with the eigenvalues $u \pm a$ (shock or rarefaction wave). A shock is produced if and only if the central state satisfies the condition

$$
\left|u_{R} \pm a_{R}\right|<\left|u_{*} \pm a_{*}\right|<\left|u_{L} \pm a_{L}\right|
$$




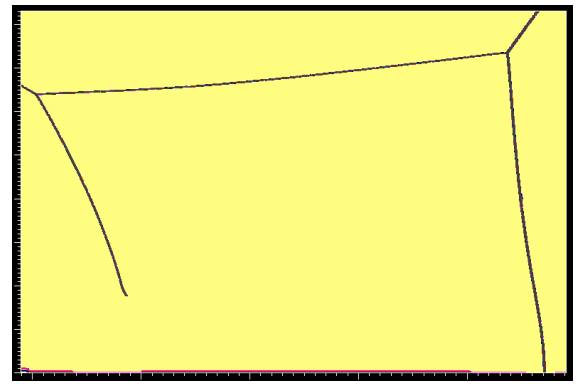

(a) Shock Detection

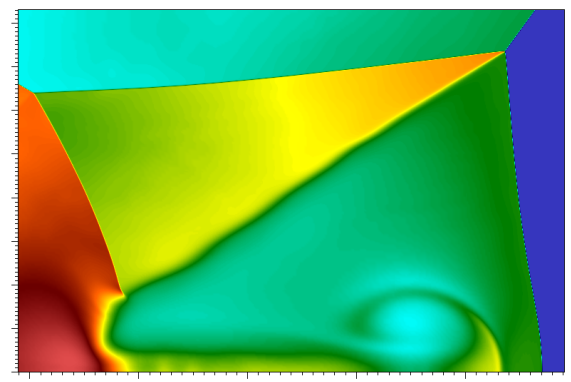

(b) Density pseudo-color plot

Figure 2: Shock detection applied to the viscous double Mach reflection problem of Section 7.1.

Here, $a_{L, R}$ is computed by evaluating the speed of sound, $a=\sqrt{\gamma p / \rho}$, at the left or right cell faces, and the central state $\left(u_{*}, a_{*}\right)$ corresponds to the Roe averages,

$$
u_{*}=\frac{\sqrt{\rho_{L} u_{L}}+\sqrt{\rho_{R} u_{R}}}{\sqrt{\rho_{L}}+\sqrt{\rho_{R}}}, \quad a_{*}=\sqrt{\left(\gamma_{*}-1\right)\left(h_{*}-\frac{1}{2} u_{*}^{2}\right)},
$$

where

$$
h_{*}=\frac{\sqrt{\rho_{L} h_{L}}+\sqrt{\rho_{R} h_{R}}}{\sqrt{\rho_{L}}+\sqrt{\rho_{R}}}, \quad c_{p, *}=\frac{\sqrt{\rho_{L} c_{p, L}}+\sqrt{\rho_{R} c_{p, R}}}{\sqrt{\rho_{L}}+\sqrt{\rho_{R}}}, \quad \gamma_{*}=\frac{c_{p, *}}{c_{p, *}-R, *}, \quad R_{*}=\frac{\sqrt{\rho_{L} R_{L}}+\sqrt{\rho_{R} R_{R}}}{\sqrt{\rho_{L}}+\sqrt{\rho_{R}}}
$$

and $h_{*}, \gamma_{*}, c_{p, *}$, and $R_{*}$ are the Roe-averaged specific enthalpy, heat ratio, specific heat for constant pressure, and gas constant, respectively. When testing the validity of the inequalities (31), a threshold value $\alpha_{\text {Liu }} / a$ is considered to eliminate weak acoustic waves that could be easily handled by the CD scheme. For better efficiency and flexibility, this criterion is combined with a geometrical test based on a mapping of the normalized pressure gradient, $\theta_{j}$, that reads $[49]$

$$
\phi\left(\theta_{j}\right)=\frac{2 \theta_{j}}{\left(1+\theta_{j}\right)^{2}} \quad \text { with } \quad \theta_{j}=\frac{\left|p_{j+1}-p_{j}\right|}{\left|p_{j+1}+p_{j}\right|} .
$$

If Equation (31) is satisfied for a cell wall bounded by cells $j$ and $j+1$ with values different by at least $\alpha_{L i u} / a$ and also $\phi\left(\theta_{j}\right)>\alpha_{\text {Map }}$ holds true, then WENO is set to be used at the cell wall. This algorithm is applied independently in each spatial direction and we additionally employ it in multiple rotated frames of reference. The latter allows us to efficiently detect shocks that are not grid aligned. In our two-dimensional simulations, to be discussed below, we use the shock-detection criterion also for rotational angles \pm 45 and \pm 135 degrees to capture shock waves perpendicular to the diagonal directions of the mesh.

\subsection{Higher-order accurate hybrid prolongation and restriction}

Prolongation involves the interpolation of cell-centered vector of state variables at a coarse level to the next finest level's ghost or newly refined interior cells. Restriction involves the interpolation (for finite difference methods) or just simple averaging (for finite volume methods) of the fine level states onto the underlying coarse level mesh. We have extended the prolongation and restriction operators commonly used (cf. [6]) from first-order to 5th-order accuracy. In order to construct the interpolation stencils, the Aitken method [55], based on Lagrange interpolation, is used sequentially in each spatial direction. The coefficients are calculated recursively allowing for different refinement factors, for example 2, 4, or 8 times finer grids. Wherever possible, centered stencils are selected as shown in Figures 3(a) and 3(b). Exactly centered or slightly upwinded (by half a cell width) stencils are used in most cases. For coarse cells adjacent to mesh boundaries, as in Figure 3(c), a stencil upwinded by one cell is required, which is a result of having 6-point 5th-order accurate stencils when 6 ghost cells are available. With our implementation, owing to the treatment of the time step stability criterion as described in the Appendix B, WENO is not needed at coarse/fine SAMR boundaries for stability. The hybrid prolongation and restriction operators have been applied successfully both with reactive and non-reactive simulations using the WENO/CD method and the shock-based discontinuity detection. With the 5th-order accurate operators, overall 6th-order convergence was found in the two-dimensional verification problems which had smooth flow. At present, these operators are unconstrained and permit local conservation errors within the order of accuracy of the used interpolation. We note that where a discontinuity is flagged, for example with the shock-based detection, the operator defaults to the stable first-order accurate interpolation. One can view 


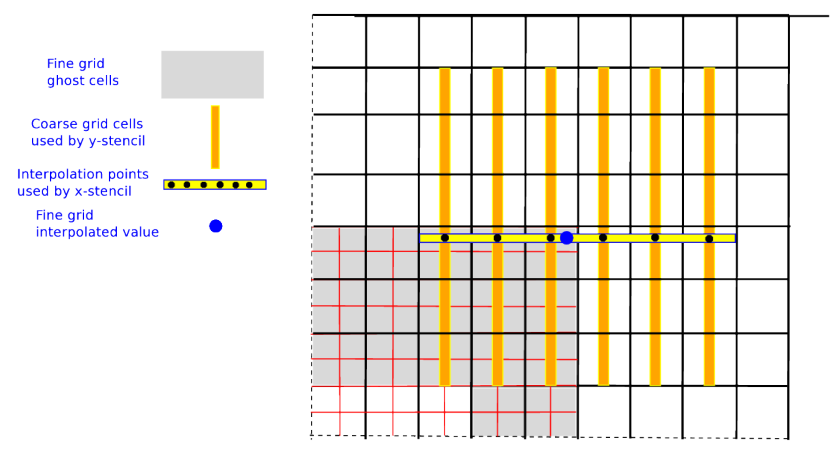

(a) Prolongation: coarse grid to fine ghost cells, upwinded

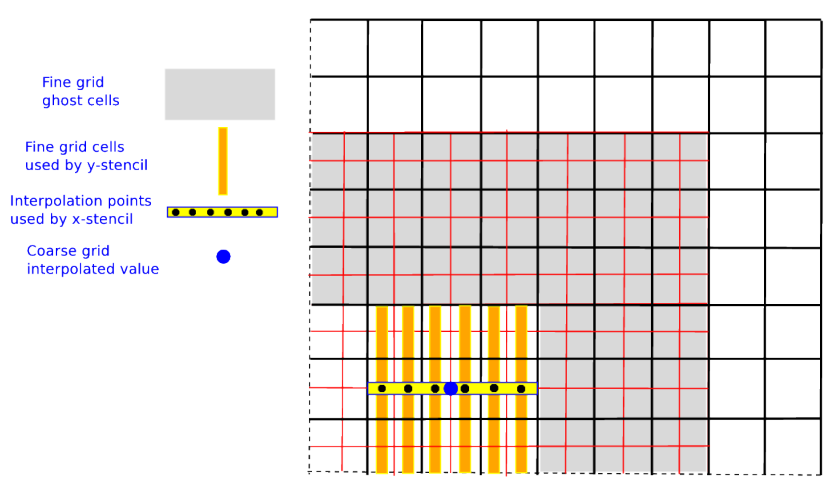

(b) Restriction: fine to coarse grid underlying cells, centered

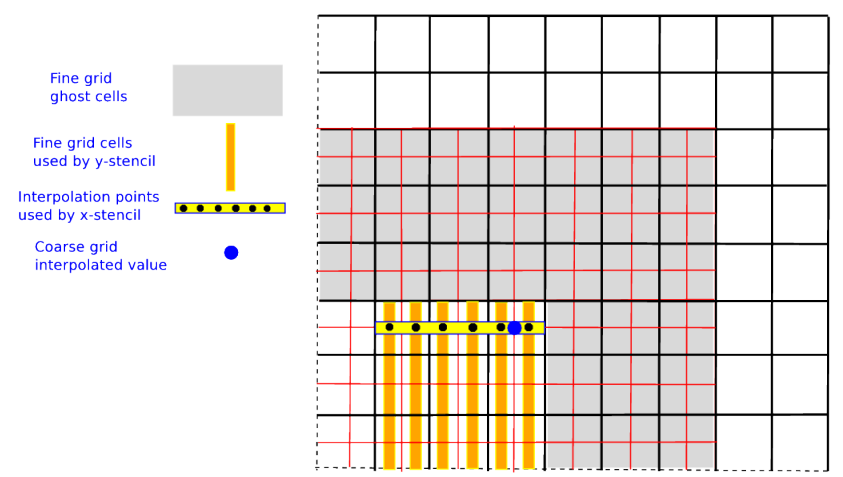

(c) Restriction: upwinded case

Figure 3: Fifth-order accurate stencils used by the hybrid-order prolongation and restriction. These stencils are used when non-discontinuous flow is encountered. Note, that all stencils are centered except for the boundary coarse grid cells set in the restriction operation.

this method as a simplified version of mesh interface "WENO" interpolation. In this respect, by making the WENO smoothness measures accessible to the prolongation and restriction functions, 5th-order fully upwinded stencils could be used at the shocks. However, in our case the option of defaulting to first-order accurate interpolation at discontinuities is chosen for simplicity.

\subsection{Multicomponent chemistry solver}

The detailed multicomponent chemistry and transport are implemented through the use of the CHEMKIN-II library [39]. This chemical kinetics package is utilized to evaluate the reaction rates, enthalpies, specific heats, and transport coefficients according to a particular reaction mechanism and thermodynamic model. The temperature is found by applying a standard Newton method to an implicit temperature equation. If the Newton method does not converge in a reasonable number of iterations, a standard bisection technique is applied. The bisection method is always guaranteed to converge [19]. In order to speed up the evaluation of temperature-dependent specific heats and enthalpies, two constant tables are constructed for each species during the start-up of the computational code [20].

\section{Verification}

A systematic verification study of the described hybrid WENO/CD method within an SAMR framework has been conducted. The presentation starts with non-reacting and reacting diffusive perfect gas flows in one space dimensions, and considers some two-dimensional benchmarks subsequently.

\subsection{One-dimensional viscous shock}

An analytical one-dimensional solution of a stationary viscous shock profile was used to verify the implementation separately in the $x$ - and $y$-direction. The analytical solution is formulated in nondimensional form, where the upstream density, pressure, and velocity (indexed with 0) in addition to an equivalent perfect gas mean free path are used as scaling parameters. See Kramer [43] for the implicit solution (with Prandtl number of $\frac{3}{4}$ and $\gamma=1.4$ ), which is expressed as a function of the Mach number and specific heat ratio, and relates the nondimensional velocity and position. The 


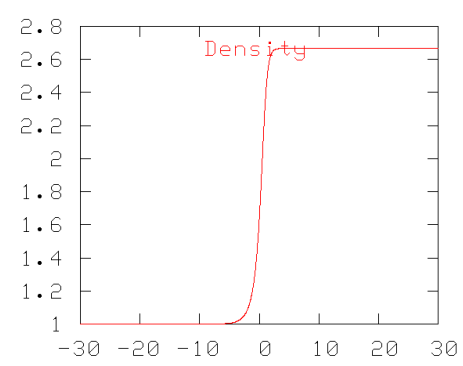

(a) Density

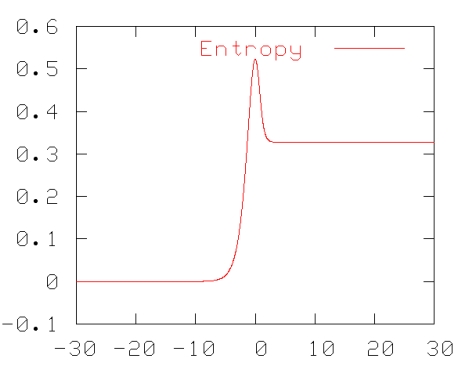

(b) Entropy

Figure 4: Analytical and numerical solution for the one-dimensional steady shock wave (visually no difference).

\begin{tabular}{|c|c|c|c|c|c|c|}
\hline Cells & $\begin{array}{c}\text { Density } \\
L_{1} \text {-error }\end{array}$ & rate & $\begin{array}{c}\text { Momentum } \\
L_{1} \text {-error }\end{array}$ & rate & $\begin{array}{c}\text { Total energy } \\
L_{1} \text {-error }\end{array}$ & rate \\
\hline Pure CD & & & & & & \\
\hline 256 & $2.31 \mathrm{E}-6$ & & $1.71 \mathrm{E}-5$ & & $1.62 \mathrm{E}-5$ & \\
\hline 512 & $7.08 \mathrm{E}-7$ & 1.71 & $2.73 \mathrm{E}-7$ & 5.97 & $3.36 \mathrm{E}-6$ & 2.27 \\
\hline 1024 & $1.14 \mathrm{E}-8$ & 5.96 & $4.38 \mathrm{E}-9$ & 5.96 & $5.44 \mathrm{E}-8$ & 5.95 \\
\hline 2048 & $1.81 \mathrm{E}-10$ & 5.98 & $6.94 \mathrm{E}-11$ & 5.98 & $8.59 \mathrm{E}-10$ & 5.99 \\
\hline Pure WENO & & & & & & \\
\hline 256 & $2.78 \mathrm{E}-5$ & & $4.01 \mathrm{E}-5$ & & $1.41 \mathrm{E}-4$ & \\
\hline 512 & $1.03 \mathrm{E}-5$ & 1.43 & $8.49 \mathrm{E}-6$ & 2.24 & $4.45 \mathrm{E}-5$ & 1.66 \\
\hline 1024 & $2.36 \mathrm{E}-7$ & 5.45 & $2.09 \mathrm{E}-7$ & 5.34 & $1.03 \mathrm{E}-6$ & 5.43 \\
\hline 2048 & $5.19 \mathrm{E}-9$ & 5.51 & $1.75 \mathrm{E}-8$ & 3.58 & $2.52 \mathrm{E}-8$ & 5.35 \\
\hline
\end{tabular}

Table 1: $L_{1}$-error norms for the three state variables of the viscous shock test problem. For the pure WENO method the $\epsilon$ value, cf. Equation (15), was set to $10^{-4}$.

specific mean free path, if only used as a length scale, can be arbitrary. For the adopted solution it is $\lambda_{0}=\frac{8 \mu}{5} \sqrt{\frac{2}{\pi \rho_{0} p_{0}}}$. The density, pressure, and hence the total energy are found with the relations,

$$
\rho u=\rho_{0} u_{0}, \quad \bar{u}=\frac{u}{u_{0}}, \quad \frac{p}{p_{0}}=\frac{\left.\left.-M^{2}\left((\gamma-1) \bar{u}^{2}-(\gamma-1)\right)\right)-2\right)}{2 \bar{u}} .
$$

By using the analytical solution as an initial condition, the Navier-Stokes equations were marched forward in time until the computation reached a stationary state. Typical shock profiles are shown in Figure 4, where exact and numerical solutions are indistinguishable. The $L_{1}$-error norm of the difference between the exact and the numerical solution was then used to verify 6th-order convergence of WENO and CD solvers, with viscous and heat conduction terms, for a perfect gas. Since this test case has a smooth solution, the WENO and CD methods were tested separately. The low convergence rates at coarse resolution (cf. Table 1) are the result of an insufficient number of points spanning the shock thickness, and the WENO and CD stencils (6 points wide), but particularly, the viscous flux stencils (12 points wide) show degraded convergence. This is generally true for all possible length scales encountered in a simulation. Note that for this and all following verification studies, because finite difference rather than finite volume methods are used, cell centered rather than cell averaged values are used in convergence tests.

\subsection{One-dimensional steady laminar flame}

As a multicomponent verification of flows with chemistry, we compared our solution for a 4 -species one-step model of hydrogen-air combustion using CHEMKIN-II and the full one-dimensional reactive multicomponent Navier-Stokes equation to the approximate FreeFlame model of CANTERA ${ }^{1}$ using the same mixture transport and kinetic model. The flame velocity and temperature were matched to that of a typical hydrogen-air flame by changing the heat release and one-step Arrhenius reaction rate and activation energy. Physical transport properties were used for the $\mathrm{H}_{2} \mathrm{O}, \mathrm{N}_{2}, \mathrm{H}_{2}, \mathrm{O}_{2}$ mixture.

The small discrepancies in the compared solutions are expected and interpreted as the result of CANTERA using a constant pressure assumption in its solution. Since the CANTERA solution is not exact for the momentum equation, there are slight differences in the steady state results to the full diffusive Navier-Stokes equations of AMROC. For our

\footnotetext{
${ }^{1}$ http://www.cantera.org
} 

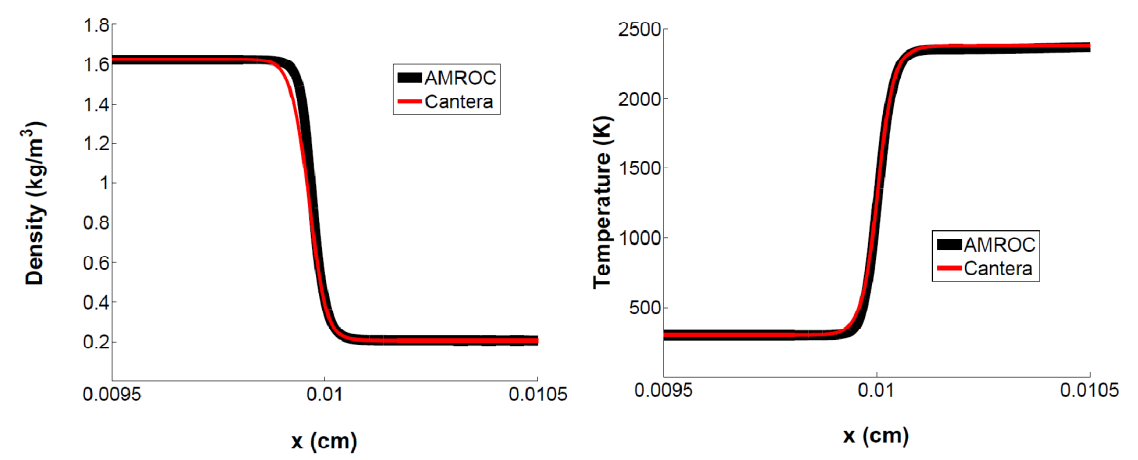

Figure 5: Multicomponent laminar flame with detailed transport. Comparison is with the steady solution of CANTERA.

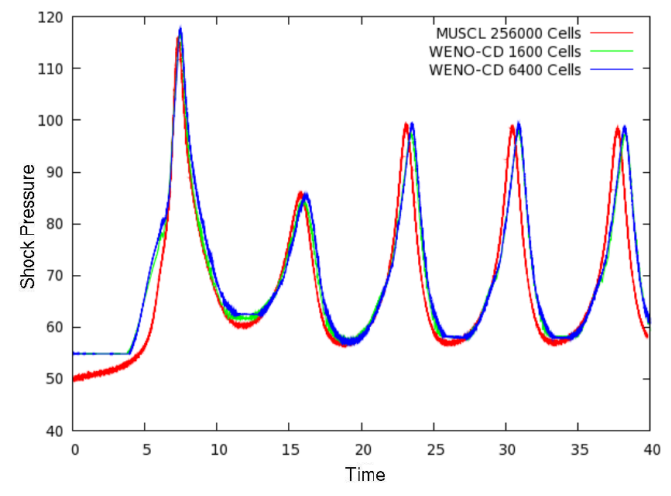

(a) Uniform grid

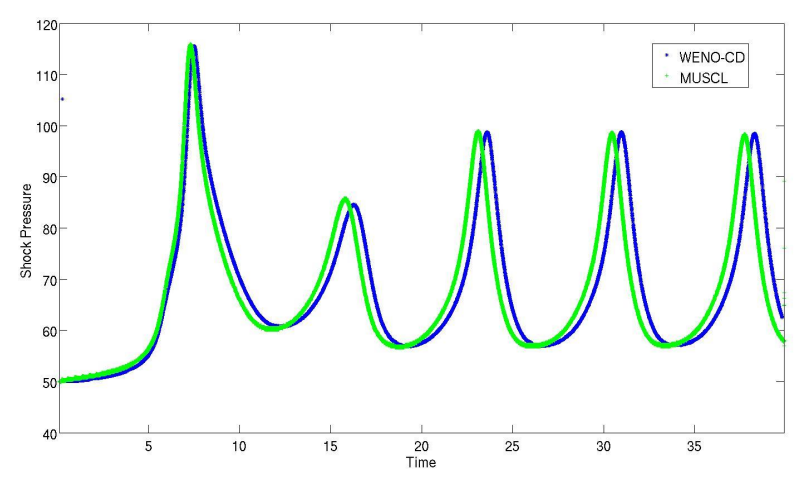

(b) 4 levels of SAMR

Figure 6: Maximum shock pressure versus time. Uniform grid: Compares the WENO/CD solutions on a grid of 1600 and 6400 cells to a MUSCL solution with 16000 cells. 4 levels: Comparing a WENO solution with 4 refinement levels (2,2,2,2) and a base grid of 1600 cells to the highly resolved MUSCL solution with 16000 cells. In each case the domain size is 80 and the final time is 40 .

AMROC solution, the CANTERA result is used as the initial condition and the solution undergoes a transient process (as the pressure adjusts) to reach a slightly different steady state. To the authors' best knowledge, there exist no analytical or semi-analytical, implicit solutions for multicomponent, reacting, diffusive flow. The difficulty is primarily due to modeling both mass diffusion and pressure gradients. Therefore, a comparison to CANTERA's widely accepted approximate flame solution was warranted.

\subsection{One-dimensional unsteady detonation}

An unsteady reactive one-dimensional problem was used to verify the interaction of the time-split chemistry terms with the reactive fluid solver. A standard unsteady detonation problem with specific heat $\gamma=1.2$, heat release $q=50$, activation energy $E=50$, gas constant $R=1$, reaction rate coefficient $A=230.75$, and overdrive $f=1.6$ is adopted to compare to the single mode period solutions found in [35] and [19]. The initial condition is the same as that used by Hwang et al. [35], using a discontinuous ambient and post-detonation state. Deiterding [19] used the steady Zel'dovich, von Neumann, and Doering (ZND) solution as the initial condition, yet, it was found that for the WENO method, the post-detonation solution is better suited as the initial non-periodic solution decays faster. The shock pressure was determined two ways, first using a local maximum, and alternatively by detecting the shock position. Because shock-capturing, rather than shock-fitting, is used, there are oscillations in the shock pressure owing to the unavoidable errors of the shock moving back and forth across the grid (when the reference frame is determined by the known average shock velocity). The best results were found by detecting the time-dependent shock position and using this to calculate the pressure, which depends analytically on the shock velocity. This shock velocity was found by using second-order differentiation of the data points corresponding to the position of the local shock pressure peaks. Using this indirect extrapolation of the velocity leads to smoother data in Figure 7 [24]. This procedure has typically not be used by previous sources.

As shown in Figure 6(a), after the initial transient relaxation, a periodic solution is reached. The features of interest, i.e., the period and amplitude of the pressure oscillation, agree, although the relaxation period is slightly 


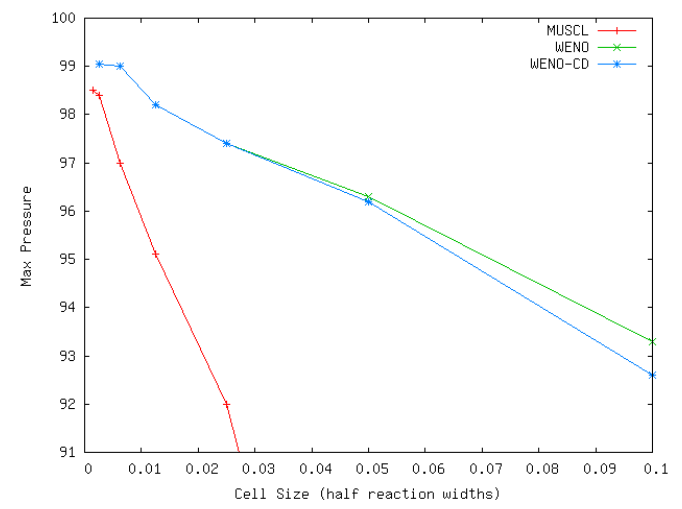

Figure 7: Convergence of the maximum pressure peak of $\sim 99$ for the WENO/CD, WENO, and MUSCL methods.

\begin{tabular}{|c|c|c|c|c|c|}
\hline $\begin{array}{c}\text { Cell Size } \\
\text { (half reaction widths) }\end{array}$ & $L_{\frac{1}{2}}$ & Cells & $\begin{array}{c}\text { MUSCL } \\
\text { Max Pressure }\end{array}$ & $\begin{array}{c}\text { WENO } \\
\text { Max Pressure }\end{array}$ & $\begin{array}{c}\text { WENOCD } \\
\text { Max Pressure }\end{array}$ \\
\hline $1.00 \mathrm{E}-001$ & 10 & 160 & - & 93.3 & 92.6 \\
\hline $5.00 \mathrm{E}-002$ & 20 & 800 & 80.9 & 96.3 & 96.2 \\
\hline $2.50 \mathrm{E}-002$ & 40 & 1600 & 92.0 & 97.4 & 97.4 \\
\hline $1.25 \mathrm{E}-002$ & 80 & 3200 & 95.1 & 98.2 & 98.2 \\
\hline $6.25 \mathrm{E}-003$ & 160 & 6400 & 97.0 & 99.0 & 99.0 \\
\hline $2.50 \mathrm{E}-003$ & 400 & 16000 & 98.4 & - & 99.05 \\
\hline $1.56 \mathrm{E}-003$ & 641 & 25600 & 98.5 & - & - \\
\hline
\end{tabular}

Table 2: Convergence of the maximum pressure peak for the one-dimensional, unstable two-species detonation problem. Values are shown for the MUSCL, WENO, and WENO/CD methods. The solution accepted by the detonation research community is 99 [19].

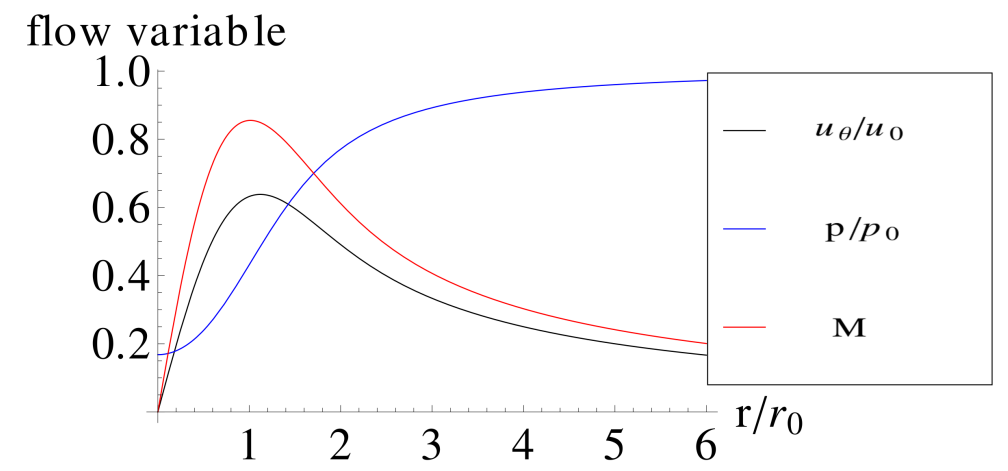

Figure 8: One-dimensional inviscid exact solution for the manufactured Lamb-Oseen vortex problem.

different for each method. The magnitude and development of the initial start-up errors are grid resolution and method dependent, which is characteristic for this unstable problem [19]. For a fairly coarse uniform grid, 20 and 80 cells per half-reaction-zone length, $L_{\frac{1}{2}}$, the $\mathrm{WENO} /$ centered difference hybrid scheme is fairly close and converges to a highly resolved MUSCL solution with 200 cells per $L_{\frac{1}{2}}$. In these tests a domain of size 80 was used. In Figure 6(b), the dynamic mesh adaptation is tested by using a WENO solution with a base grid of 20 cells per $L_{\frac{1}{2}}$ along with 4 refinement levels, which corresponds to 320 cells per $L_{\frac{1}{2}}$ near the shock front.

In Figure 7 and Table 2, the convergence of the WENO/CD and pure WENO was compared in the uniform grid case to the convergence of the widely accepted MUSCL method as used by Deiterding [19]. The method of Hwang et al. [35], a 3rd-order WENO scheme, also converges to a pressure of approximately 99. Here it is found that the WENO and WENO/CD solutions converge at similar rates to the maximum shock pressure. As expected, this is substantially higher than that of the 2nd-order MUSCL solution at the same resolution. Note that at least 10 cells per $L_{\frac{1}{2}}$ are required to have an acceptable maximal pressure amplitude and period. 


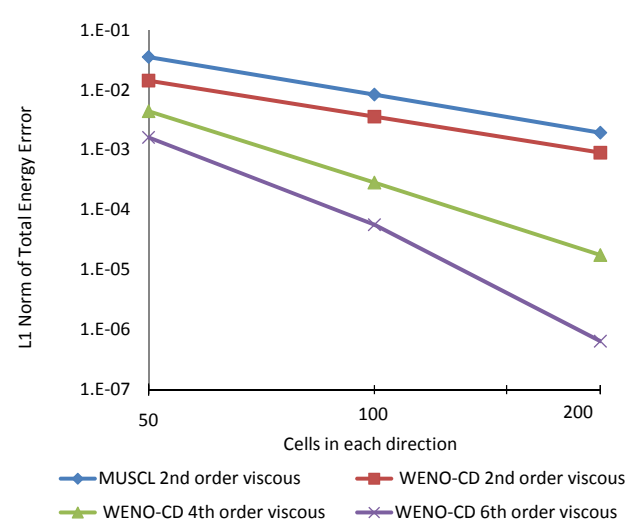

(a) Uniform mesh

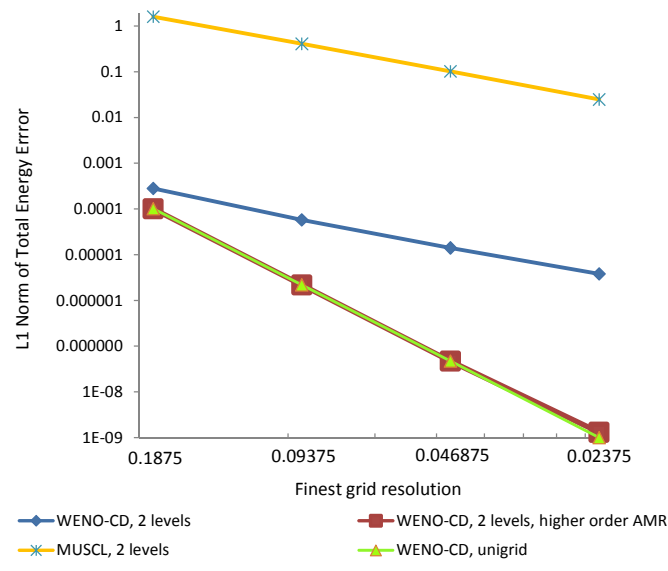

(b) Two SAMR levels

Figure 9: Convergence plot (log-log scale) for the manufactured Lamb-Oseen vortex solution (a) and the viscous decaying vortex (b). The convergence plots show the decrease of the $L_{1}$-error norm of the total energy as the resolution is doubled.

\begin{tabular}{|c|c|c|c|c|c|c|c|c|}
\hline Cells & $\begin{array}{c}\text { MUSCL } \\
\text { 2nd order } \\
\text { viscous } \\
L_{1} \text {-error }\end{array}$ & rate & $\begin{array}{c}\text { WENO/CD } \\
\text { 2nd order } \\
\text { viscous } \\
L_{1} \text {-error }\end{array}$ & rate & $\begin{array}{c}\text { WENO/CD } \\
4 \text { th order } \\
\text { viscous } \\
L_{1} \text {-error }\end{array}$ & rate & $\begin{array}{c}\text { WENO/CD } \\
6 \text { th order } \\
\text { viscous } \\
L_{1} \text {-error }\end{array}$ & $\begin{array}{c}\text { rate } \\
\text { rater }\end{array}$ \\
\hline $100^{2}$ & 0.0355 & & 0.0143 & & 0.00442 & & 0.00162 & $5.65 \mathrm{E}-05$ \\
\hline $200^{2}$ & 0.00839 & 2.08 & 0.00359 & 1.99 & 0.000284 & 3.96 & 4.84 \\
\hline
\end{tabular}

Table 3: Convergence on uniform grids using the manufactured solution for the two-dimensional Lamb-Oseen vortex test, showing the error values of the $L_{1}$-norm of the total energy and the corresponding convergence rates for different methods with differing viscous flux stencils.

\begin{tabular}{|c|c|c|c|c|c|c|c|c|}
\hline $\begin{array}{l}\text { Finest grid } \\
\text { resolution }\end{array}$ & $\begin{array}{c}\text { WENO/CD } \\
2 \text { levels } \\
L_{1} \text {-error }\end{array}$ & rate & $\begin{array}{c}\text { WENO/CD } \\
2 \text { levels } \\
\text { higher order SAMR } \\
L_{1} \text {-error }\end{array}$ & rate & $\begin{array}{c}\text { MUSCL } \\
2 \text { levels } \\
L_{1} \text {-error }\end{array}$ & rate & $\begin{array}{c}\text { WENO/CD } \\
\text { unigrid } \\
L_{1} \text {-error }\end{array}$ & rate \\
\hline 0.1875 & 0.000282 & & 0.000101 & & 1.60 & & 0.000102 & \\
\hline 0.09375 & $5.80 \mathrm{E}-05$ & 2.28 & $2.18 \mathrm{E}-06$ & 5.53 & 0.409 & 1.97 & $2.19 \mathrm{E}-06$ & 5.54 \\
\hline 0.046875 & $1.40 \mathrm{E}-05$ & 2.05 & $4.72 \mathrm{E}-08$ & 5.53 & 0.103 & 1.99 & $4.75 \mathrm{E}-08$ & 5.53 \\
\hline 0.0234375 & $3.82 \mathrm{E}-06$ & 1.87 & $1.31 \mathrm{E}-09$ & 5.17 & 0.0248 & 2.05 & $1.01 \mathrm{E}-9$ & 5.56 \\
\hline
\end{tabular}

Table 4: Convergence results for the decaying (viscous) two-dimensional Lamb-Oseen vortex benchmark, showing the error values of the $L_{1}$-norm of the total energy and the corresponding convergence rates. Except for the MUSCL method, each WENO/CD test case uses 6 th-order accurate viscous flux stencils, yet only the uniform grid and the multi-level test case using higher-order prolongation and restriction yields close to 6th-order convergence.

\subsection{Two-dimensional manufactured and decaying Lamb-Oseen vortex}

For verification of the convergence properties of both the inviscid and viscous fluxes, a two-dimensional "manufactured solution" of the convecting Lamb-Oseen vortex [62] was constructed. Radial profiles for this exact solution, shown in Figure 8, were obtained for a steady, inviscid vortex problem. An exact viscous, steady solution was then constructed by adding viscous fluxes together with analytically known source terms in both the momentum and energy equations to cancel them exactly. An order-verification via the manufactured solution procedure (OVMSP) [42] creates analytic solutions to the fully general differential equations solved by the code and uses a grid convergence study to confirm the order of accuracy. Fourth- and sixth-order, conservative (in each SAMR level) viscous fluxes were constructed and verified. A convergence study was conducted for a uniform and a two-level grid. When using first-order accurate prolongation and restriction, global 3rd-order spatial convergence was found for the two-level grid over a finite time for the 6th- and 4th-order methods. The uniform grid convergence results are shown in Figure 9 (a) and Table 3.

Further, a separate verification problem was constructed using a highly accurate, but approximate solution of the convecting, viscous, decaying Lamb-Oseen vortex. Here, the expected 6th-order convergence rate using the 5th-order prolongation and restriction was verified using the full compressible Navier-Stokes equations. For these test cases, a highly resolved $4096 \times 4096$ mesh was used as reference result, and was compared to base meshes of $64 \times 64$ to $512 \times 512$ cells. This convergence test was carried out using two levels, a base grid with a static $2 \times 2$ refinement mesh, centered in 


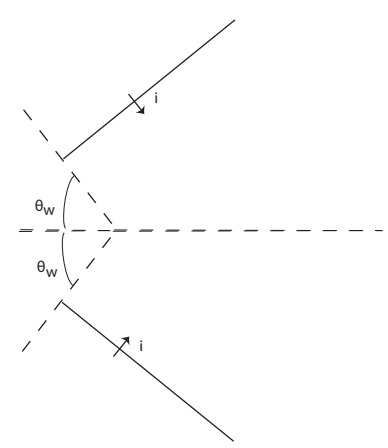

(a) Initial State

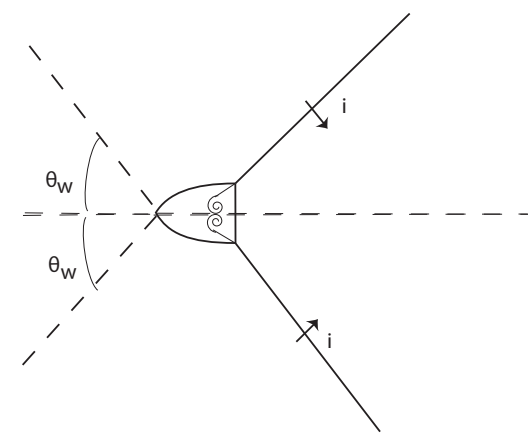

(b) $t>0$
Figure 10: The model problem of two interacting planar shock waves.

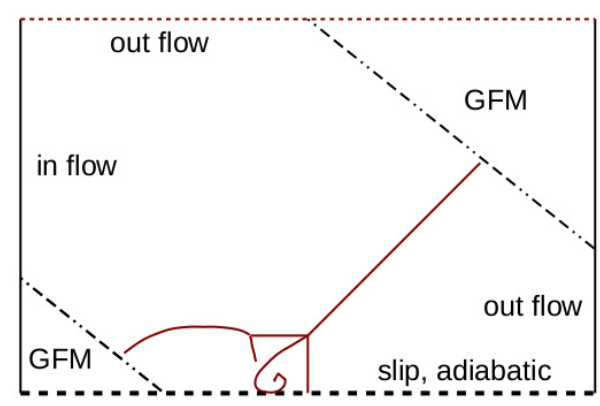

Figure 11: Boundary conditions for the reactive double Mach reflection problem. For the non-reactive problem, the top ghost-fluid-method (GFM) region is not needed and the exact shock solution is used at the upper boundary.

the vortex. In Figure 9(b) and Table 4, with 2 levels, 2nd-order convergence is found for all methods using the standard 1st-order accurate prolongation and restriction operators. With a uniform grid, 6th-order convergence is confirmed for WENO/CD. Lastly, with the new 5th-order accurate hybrid prolongation and restriction, overall 6th-order convergence is achieved even for the SAMR results. Note that in the SAMR case, the error is evaluated as the sum of the $L_{1}$-error norms on the domain $\Omega_{\lambda}$ of level $\lambda$ without higher refinement. Denoting by $\Lambda$ the highest level available, the norm calculation reads

$$
L_{1}(q)=L_{1}^{e}\left(\Delta x_{\Lambda}, \Delta y_{\Lambda}, \Omega_{\Lambda}\right)+\sum_{\lambda=0}^{\Lambda-1} L_{1}\left(\Delta x_{\lambda}, \Delta y_{\lambda}, \Omega_{\lambda} \backslash \Omega_{\lambda+1}\right) \text {, }
$$

where

$$
L_{1}(\Delta x, \Delta y, \Omega)=\sum_{j, l}\left|q_{j l}-q_{j l}^{r}\right| \Delta x \Delta y
$$

is the $L_{1}$-error norm on the domain $\Omega$, and where $q_{j l}^{r}$ denotes the averaging projection of the reference solution from the $4096 \times 4096$ uniform mesh down to the desired mesh with step sizes $\Delta x, \Delta y$.

\section{Shock-driven combustion}

A canonical problem for shock-induced combustion in compressible flow is the detonation wave. A multidimensional detonation wave is described as having a quasi-periodic structure of transverse waves in addition to the leading shockreaction front. A key repetitive sub-structure within gaseous detonations is the three-shock or triple point configuration that arises due to a fundamental instability. Here, Mach stems and incident shocks are part of the leading shock and the resulting reflected shocks are the transverse waves. However, Mach reflection structures can also be created when shock waves encounter solid boundaries. In the following, we will concentrate on obtaining an accurate DNS simulation of just this fundamental triple point structure.

\subsection{The model problem}

Detonations are intrinsically three-dimensional; however, due to the complexity of the problem and the ability to easily extend an implementation from two to three space dimensional, a two-dimensional model is used. The problem setup for a two-dimensional, shock-initiated detonation reflection is depicted schematically in Figure 10(a). Two detonation waves travel symmetrically down inclined wedges of equal angle $\theta_{w}$ and then collide. As depicted schematically in Figure 10(b), the collision produces a symmetrical structure consisting of two triple points with Mach stems, reflected waves, and shear layers, one set for each incident shock wave. To reduce computational cost, the symmetry of the problem is utilized. For our preliminary results, one non-reacting perfect gas species with constant specific heat was used. Later, one-step and multi-step reaction models, involving multiple species, were employed. Note that a Reynolds number Re for this flow can be defined by using the ambient density, speed of sound and also the distance the incident shock has traveled along the bottom boundary,

$$
\operatorname{Re}=\frac{\rho_{\infty} a_{\infty} L(t)}{\mu_{\infty}}, \quad L(t)=d_{\text {shock }} \sin \theta_{w} t
$$




\subsection{Length scales and resolution}

In order to achieve accurate numerical simulation results, all physically relevant length and time scales must be resolved, regardless of the order of accuracy of the numerical method. For our method, having at least 10 to $10^{2}$ cells across a length scale is required before one can begin testing for convergence. The detonation problem has multiple length scales, which requires care both in setting up an adaptive solution and in interpreting the results. The incident shock thickness is slightly larger than the nondimensional length scale used in the exact one-dimensional viscous shock solution. The shock thickness reads

$$
\lambda_{\text {shock }}=\frac{8 \mu(T)}{5} \sqrt{\frac{2}{\pi \rho_{\infty} p_{\infty}}} .
$$

It is of the order of three to five mean free path lengths. Note that in a Mach reflection pattern, the smallest shock thickness is found in the Mach stem. Presently, we do not resolve this length scale and in this sense we are performing pseudo-DNS. With shock-capturing, numerical dissipation will smear the shock in this region, yet, the other areas of the flow field are largely unaffected if their other scales (reactive and viscous) are resolved. The diffusive length scales, which include viscosity, heat, and mass diffusion, are most prevalent in the shear layer. On dimensional grounds, the viscous shear layer thickness, $\delta_{\text {visc }}$, grows as

$$
\delta_{\mathrm{visc}} \approx \sqrt{\nu t}
$$

where $\nu=\frac{\mu}{\rho}$ is the dynamic viscosity, while the thermal heat conduction layer thickness, $\delta_{\text {cond }}$, and the mass diffusion layer thickness, $\delta_{\text {mass }, i}$, grow as

$$
\delta_{\mathrm{cond}} \approx \sqrt{\frac{k_{\mathrm{ref}} t}{\rho c_{v}}}, \quad \delta_{\mathrm{mass}, i} \approx \sqrt{\frac{D_{i}}{\rho} t},
$$

respectively. For hydrogen and hydrocarbon fueled detonations, typically the smallest scale is the viscous shear thickness, followed by the mass, and then the heat diffusion thickness.

Also, for the chemical reactions, there are two different types of scales. A fundamental time scale comes from each Arrhenius rate equation and for a simple one-step model is

$$
\tau_{\mathrm{Arr}}=\frac{1}{A Y_{1} T^{n}} \exp \left(\frac{E_{a}}{R T}\right) .
$$

For a mixture with multiple reactions, associated time scales can differ by orders of magnitude. A further length scale of larger size is the laminar flame thickness, which results from the interaction of the chemical rate equations, diffusion, heat conduction, and convection. Thus far, we only run simulations for time scales capturing the flames in the laminar regime. Therefore, for the results presented, we check the resolution of the smallest length scale, the viscous shear layer thickness.

\subsection{Initial and boundary conditions}

For the non-reactive simulations, the setup involves pre- and post-shock initial conditions throughout the domain. The boundary conditions include vanishing normal velocity, tangential stress and heat conduction on the inclined portions of the "wedge"; symmetry boundary conditions on the horizontal boundary with zero normal velocity, tangential stress and heat conduction on the horizontal boundary, and simple zeroth-order inflow/outflow boundary conditions on the left and right boundaries. Since this is a compressible flow, the boundary errors do not travel fast enough to interact with the solution area of interest. The inclined slip surface boundary is enforced using the level set implementation of the first-order accurate ghost fluid method [17]. The exact traveling shock solution is prescribed along the top boundary. For this non-reactive case, the setup is similar geometrically to the wedge interaction problem studied by Vas Ilev et al. [36]. We use, however, different boundary conditions as our present interest is in shock-shock interactions rather than shock-solid boundary interactions. For the reactive case, the one-dimensional or planar ZND detonation wave solution was used as initial condition. This admits finite-rate chemical reactions and describes a detonation as an infinitely thin shock wave followed by a zone of exothermic chemical reaction. The shock travels with a speed given by the Chapman-Jouguet condition. The initial condition is found by numerically solving the one-dimensional, steady, reactive Euler equations using a numerical ordinary differential equation (ODE) solver. Also, in order to reduce boundary errors, the top boundary was angled and set to a solid slip boundary condition, as shown in Figure 11. The initial and boundary conditions for the multicomponent, detailed chemistry results are the same as in the two-component, one-step chemistry case, aside from a differing ZND solution due to the increased complexity in chemistry. 


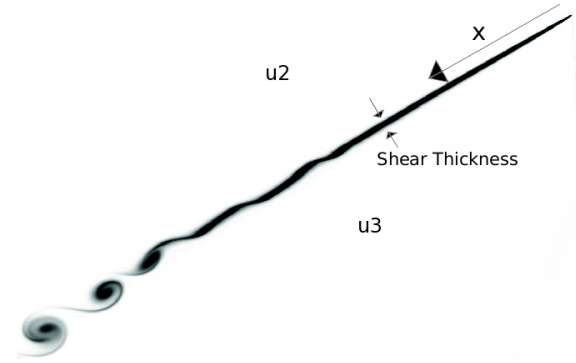

(a) Vorticity in the mixing layer

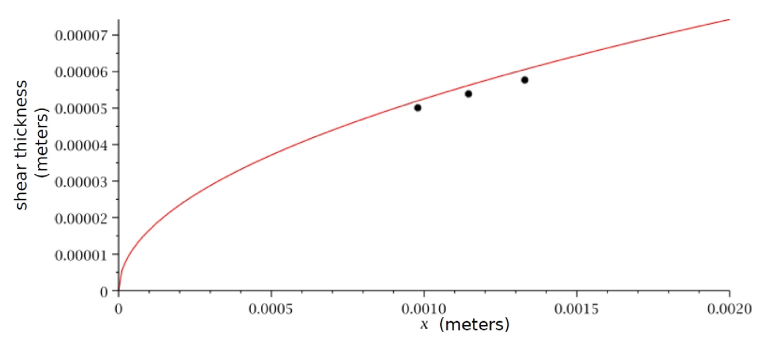

(b) Mixing thickness growth

Figure 12: Vorticity in the mixing layer and the laminar mixing layer thickness as a function of distance from the triple point, von Karman momentum-integral technique. The last black dot is from our numerical simulation for the approximate thickness at a distance of $1.35 \mathrm{~mm}$ behind the triple point. This thickness is in the laminar stable regime.

\section{Applications}

\subsection{Non-reactive diffusive double Mach reflection}

A fully resolved unsteady DMR simulation in air with $\gamma=1.4$ was conducted. The initial conditions were selected to match the numerical and experimental results of Vas Ilev et al. [36], where a Mach number $M=4.5$, inflow angle $\theta_{w}=36^{\circ}$, and viscosity and thermal conductivity of $\mu=1.73 \cdot 10^{-5} \mathrm{~Pa} \cdot \mathrm{s}$ and $k=2.57 \cdot 10^{-2} \mathrm{~W} /(\mathrm{m} \cdot \mathrm{K})$ were chosen. However, in order to gauge the performance of the code for detonation simulations, a slip boundary condition was applied, rather than the no-slip boundary condition proposed by Vas Ilev et al. [36]. The constant viscosity and conductivity correspond approximately to the average values for the post-shock conditions for the ambient state with $T=300 \mathrm{~K}$ and $p=2000 \mathrm{~Pa}$. A nondimensionalization or scaling of the fluid dynamic equations was used and is detailed in Appendix A. The maximum CFL parameter for automatic time step adjustment was 0.98 using the ten-step RK4 integration. Refinement criteria that capture the physics of each length scale in the problem were utilized. The density gradient is used to refine the convective length scale, the $x$ - and $y$-velocity gradients are used for the viscous length scale, and the energy gradient for the conduction lengths. The viscous length scale is estimated by using the average density of the top and bottom flows of the shear layer $\rho_{\text {avg }}=0.1496 \mathrm{~kg} / \mathrm{m}^{3}$. The shock speed and speed of sound used to calculate the Reynolds number are 1,566 and $348 \mathrm{~m} / \mathrm{s}$, respectively. Through experience, it has been found that for (40), using the time value at which the shear layer begins to become unstable is sufficient for calculating a viscous length scale $\delta_{\text {visc }}$. Note this naturally applies only to resolved simulations, in which there are at least 10 to 100 cells within $\delta_{\text {visc }}$ for our discretization approach.

Table 5 summarizes runs performed for the non-reactive, diffusive double Mach reflection. For the result shown in Table $5(\mathrm{D})$ the incident shock thickness (encompassing the high gradient part) is only slightly larger than $\lambda_{\text {shock }}=$ $\frac{8 \mu}{5} \sqrt{\frac{2}{\pi \rho_{\infty} p_{\infty}}} \approx 3.2 \cdot 10^{-6} \mathrm{~m}$. This corresponds to approximately 100 cells across the shear layer (at finest grid resolution) immediately behind the triple point and some 10 cells across the incident shock.

In the absence of an exact solution and with the necessity of adaptive mesh refinement to resolve the scales, a standard convergence study is difficult. For the shear-layer portions of the flow, comparisons with free shear layer theory were used to study solution accuracy. Directly behind the triple point, the flow is laminar and stable; therefore, for constant viscosity, the thin-layer equations apply. The similarity solution obeys the Blasius ODE but with boundary conditions for the free-mixing layer. A demonstrative numerical result is shown in Figure 12(a). Here, the growth and transition to instability (the initial inviscid mode) of the region dominated by vorticity is shown. The mixing thickness can also be obtained using the von Kármán momentum-integral technique [5]. It is assumed that the flows on both sides of the shear layer are incompressible and that there is no pressure gradient along the layer. As assumed in Bendor [5], the upper and lower velocities, $u_{2}$ and $u_{3}$ as shown in Figure 12(a), tangential to the interface are assumed to have a laminar profile and are approximated with third-order polynomials. The benefit of the von Kármán integral method is that it approximates the effects of the density difference across the layer, unlike the Blasius method, for which an average density was used. Of equal importance is that it also allows for a viscosity variation: the lower fluid is much hotter than the upper fluid, yielding a physically non-negligible change in viscosity. Yet, for the sake of simplicity, the total displacement thickness was calculated and shown in Figure 12(b). The dot on Figure 12(b) shows the comparison of the numerical and boundary layer theory results. In addition, the high-resolution results were compared to a simulation with one less refinement level, which supported visual convergence. 




Table 5: Non-reactive diffusive double Mach reflection: pseudo-color density and contour plots of the DMR structure. Figure (A) displays the long-term behavior and Figures (B) to (D) demonstrate the convergence of the WENO/CD method in resolving the viscous processes in the shear layer, by showing a succesive increase in resolution by a factor of 2 for three simulations. The approximate viscous scale and minimum cell size show the required resolution for convergence of the viscous DMR problem. Note: For the electronic version, be sure to use the zoom tool in the PDF reader to study the details of the high resolution plots.

\subsubsection{Convergence results}

A series of simulations were conducted to investigate the influence of resolution and SAMR level distribution on the initial roll-up of the shear layer. Pseudo-color and contour plots of the density are presented in Table 5 (B) to (D) for 3 different SAMR resolutions. Through this investigation, it was found that setting the refinement thresholds to enable adequate coverage of the shear layer and its surrounding region is vital for convergence because the interactions of the SAMR levels create grid-level disturbances that can influence the initially highly sensitive vortical roll-up. The region behind the shock wave close to the second triple point requires the highest level of refinement (used similarly for the shear layer) because the roll-up first occurs behind this shock. 


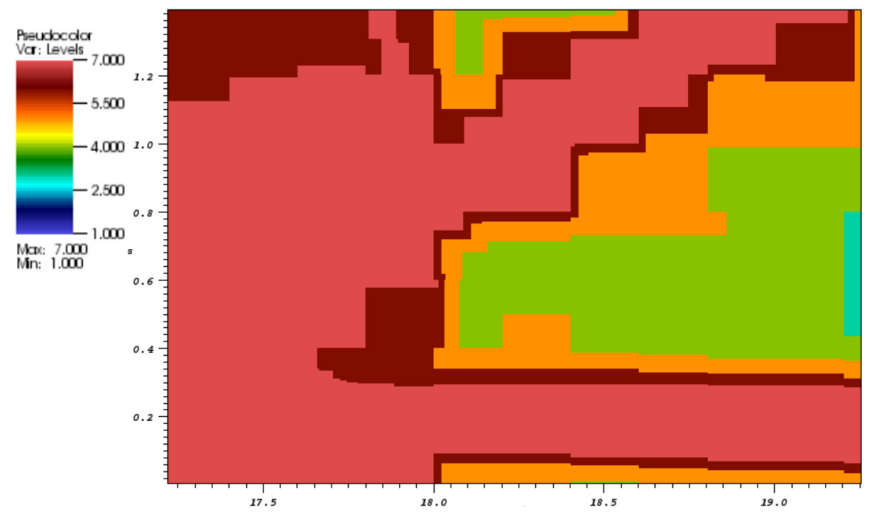

Figure 13: SAMR levels for the DMR convergence test. The domain corresponds to those of tests (B)-(D) of Table 5.

\begin{tabular}{|c|c|c|c|c|c|c|}
\hline Levels & $\begin{array}{c}\text { Density } \\
L_{1} \text {-error }\end{array}$ & rate & $\begin{array}{c}y \text {-velocity } \\
L_{1} \text {-error }\end{array}$ & rate & $\begin{array}{c}\text { Total energy } \\
L_{1} \text {-error }\end{array}$ & rate \\
\hline$t=3.48$, domain: $[17.0,24.0] \times[0.0,3.5]$ & & & & & & \\
\hline 3 & 1.05458 & - & 0.429532 & - & 31.4198 & - \\
\hline 4 & 0.675551 & 0.64 & 0.295575 & 0.54 & 20.6763 & 0.60 \\
\hline 5 & 0.304157 & 1.15 & 0.131237 & 1.17 & 9.41859 & 1.13 \\
\hline 6 & 0.223831 & 0.44 & 0.090042 & 0.54 & 6.35662 & 0.57 \\
\hline$t=3.60$ domain: $[17.0,24.0] \times[0.0,3.5]$ & & & & & & \\
\hline 3 & 1.11937 & - & 0.460977 & - & 33.0184 & - \\
\hline 4 & 0.78734 & 0.51 & 0.340686 & 0.44 & 23.912 & 0.47 \\
\hline 5 & 0.389708 & 1.01 & 0.164079 & 1.05 & 12.1556 & 0.98 \\
\hline 6 & 0.284423 & 0.45 & 0.115549 & 0.51 & 8.31585 & 0.55 \\
\hline$t=3.84$ domain: $[18.5,24.0] \times[0.0,3.5]$ & & & & & & \\
\hline (incident shock at edge of domain) & & & & & & - \\
\hline 3 & 1.24815 & - & 0.53609 & - & 37.1056 & - \\
\hline 4 & 1.07438 & 0.22 & 0.46187 & 0.21 & 32.7679 & 0.18 \\
\hline 5 & 0.576804 & 0.90 & 0.240978 & 0.94 & 17.843 & 0.88 \\
\hline 6 & 0.413381 & 0.48 & 0.175842 & 0.45 & 12.3752 & 0.53 \\
\hline
\end{tabular}

Table 6: $L_{1}$-error norms for some state variables using the 7 -level case as the reference solution.

Using the highest resolved case as an approximate "exact" numerical solution, the convergence of the WENO/CD method was quantified. As an example, Figure 13 shows the distribution of SAMR refinement levels for the 7-level case at a time of $t=3.48$. The $L_{1}$-error norms in Table 6 demonstrate the convergence of the state variables, the density, momentum, and total energy. The computation of these norms utilized only the domain surrounding the shear layer. Here, convergence is demonstrated from the time the shear layer begins to roll up, $t=3.36$, to the time at which half of the shear layer has left the domain, $t=3.84$. For these norms, rates of convergence are calculated assuming that all levels are being refined, rather than just the finest level.

Also shown is Table 7, where instead of changing the number of levels, the resolution of the base grid is halved in each direction. Here, because all grids are being refined/coarsened rather than just those coinciding with the finest grid, the convergence rates are slightly higher. Even higher rates are found when a domain excluding the shock waves is used for the norms. As an example, the rate of convergence for only the unstable jet at the bottom of the domain is shown. In this case the last rate for the density increases from 1.33 to 1.43 and for the total energy, from 1.35 to 1.44. Note that in all of these DMR test cases for Tables 6 and 7, when comparing one result to another, all the coarse/fine grid boundaries do not exactly match up. This is unavoidable with our adaptive mesh refinement strategy, as the grid is being updated at every time step and its coverage depends on the local gradients of the solution.

The evaluation of the $L_{1}$-error norms is carried out as described at the end of Section 5.4, with the only difference that the reference solution $q^{r}$ itself is now defined on a hierarchical SAMR mesh. The data on the highest locally available level of the reference solution is averaged onto the currently evaluated level of the present approximation. Since the SAMR approach leads to refinement domains $\Omega_{\lambda}$ that are always properly nested within the next coarser level domain $\Omega_{\lambda-1}$ plus a buffer region of at least one layer of coarse level cells, it is safe to assume that for the cases considered here the reference solution is always significantly better resolved. We have implemented the used error norm computation for large-scale parallel adaptive simulations as a mere post-processing step reading the I/O files generated by AMROC into a separate single-processor program. 


\begin{tabular}{|c|c|c|c|c|}
\hline Base grid & $\begin{array}{c}\text { Density } \\
L_{1} \text {-error }\end{array}$ & rate & $\begin{array}{c}\text { Total energy } \\
L_{1} \text {-error }\end{array}$ & rate \\
\hline $\begin{array}{c}t=3.84 \text { domain: }[19.2,24.0] \times[0.0,3.23] \\
\text { (incident shock at edge of domain) }\end{array}$ & & & & \\
\hline $62 \times 11$ & 1.17987 & - & 36.6927 & - \\
\hline $124 \times 22$ & 0.516237 & 1.19 & 15.7583 & 1.22 \\
\hline $248 \times 43$ & 0.289879 & 0.83 & 8.63115 & 0.87 \\
\hline $\begin{array}{c}t=3.84 \text { domain: }[19.2,23.55] \times[0.0,1.14] \\
\text { bottom jet (shocks are not included) }\end{array}$ & & & & \\
\hline $62 \times 11$ & 0.946061 & - & 29.9732 & - \\
\hline $124 \times 22$ & 0.418507 & 1.18 & 13.0559 & 1.20 \\
\hline $248 \times 43$ & 0.204751 & 1.03 & 6.27181 & 1.06 \\
\hline
\end{tabular}

Table 7: $L_{1}$-error norms for some state variables using the 7 -level case (with $496 \times 86$ base grid) as the reference solution. Coarser solutions also have 7-levels, but use coarser base grids.

These quantitative measures and visual comparisons of the solution support that our method is converging. In our two highest resolutions shown in Table 5, the diffusive processes are resolved.

\subsection{Double Mach reflection detonation}

Demonstrative, fully-resolved, diffusive, detonation-reflection simulations have also been completed in two space dimensions for a one-step, two-species, marginally stable detonation. The problem setup is similar to the non-reactive case, except for modifications of initial flow field and boundary conditions, required for an extension of a shock wave to a detonation. Simulations were conducted using the WENO/CD method and the robust MUSCL method for comparison. It was found that the accuracy of the flow was highly sensitive to the particular locations of the SAMR levels. A simple two-species, single-reaction model was used with temperature dependent transport properties. Because of the later goal of using multicomponent detailed chemistry, the thermodynamical and transport properties of our model where selected and fitted to physical parameters for a $\mathrm{H}_{2}-\mathrm{O}_{2}$ detonation initially at $T=300 \mathrm{~K}$ and $p=6,700 \mathrm{~Pa}$. Using a high-temperature extension of the GRI30 mechanism in CANTERA and the SDToolbox ${ }^{2}$, the ZND solution was calculated with detailed chemistry and used to make an approximate chemistry and transport model with a single Arrhenius rate equation, constant specific heat, and temperature dependent transport.

The Arrhenius rate activation energy and pre-exponential, heat release, and specific heat ratio were chosen by matching the Chapman-Jouguet speed and the von Neumann (post-shock) pressure at the beginning of the ZND detonation. For this two-species, calorically perfect model we have

$$
\gamma=\gamma_{1}=\gamma_{2}, p=\rho R T, R=R_{1}=R_{2} \quad \text { and } \quad \rho=\rho_{1}+\rho_{2}, \rho_{1}=\rho Y_{1}, \rho_{2}=\rho Y_{2} .
$$

With the total energy defined by the heat release per unit mass parameter, $q$, the equation of state takes the explicit form

$$
\rho e_{t}=\frac{p}{(\gamma-1)}+\frac{1}{2} \rho\left(u^{2}+v^{2}\right)+\rho_{1} q \quad \text { or } \quad e_{t}=\frac{p}{(\gamma-1) \rho}+\frac{1}{2}\left(u^{2}+v^{2}\right)+q Y_{1} .
$$

This is equivalent to having product and reactant enthalpies of the form

$$
h_{1}=h_{0}+q+c_{p} T, \quad h_{2}=h_{0}+c_{p} T \quad \Longrightarrow \quad h_{2}-h_{1}=h_{\text {prod }}-h_{\text {react }}=\Delta h_{\text {reaction }}=-q .
$$

The mass fraction production rates are

$$
\dot{\omega}_{1}=-\rho Y_{1} A \exp \left(\frac{E_{a}}{R T}\right) \quad \text { and } \quad \dot{\omega}_{2}=\rho Y_{1} A \exp \left(\frac{E_{a}}{R T}\right) .
$$

The thermodynamic parameters $T_{\infty}=300 \mathrm{~K}, p_{\infty}=6670 \mathrm{~Pa}, \rho_{\infty}=0.077552 \mathrm{~kg} / \mathrm{m}^{3}, \gamma=1.29499, W=0.029 \mathrm{~kg} / \mathrm{mol}$, $q=54000 \mathrm{~J} / \mathrm{mol}, E_{a}=30000 \mathrm{~J} / \mathrm{mol}$, and $A=6 \cdot 10^{5} \mathrm{~s}^{-1}$ were used. This corresponds to a post-shock pressure of approximately $1.7 \mathrm{~atm}$ and detonation shock speed of $d_{\text {shock }}=1587.84 \mathrm{~m} / \mathrm{s}$. The viscosity, thermal conductivity, and mass diffusions were selected by matching general trends and the values at the end of the reaction zone of the one-step and detailed chemistry models encountered in their separate one-dimensional steady ZND solutions. In all of theses ZND configurations, the solution and parameters are described as a function of position or equivalently time. For the viscosity and conductivity, the Sutherland model was used,

$$
\mu=\mu_{\text {ref }}\left(\frac{T}{T_{\text {ref }}}\right)^{\frac{3}{2}} \frac{T}{T_{\text {ref }}}, \quad k=k_{\text {ref }}\left(\frac{T}{T_{\text {ref }}}\right)^{\frac{3}{2}} \frac{T}{T_{\text {ref }}} .
$$

\footnotetext{
${ }^{2}$ http://www.galcit.caltech.edu/EDL/public/cantera/html/SD_Toolbox/index.html
} 

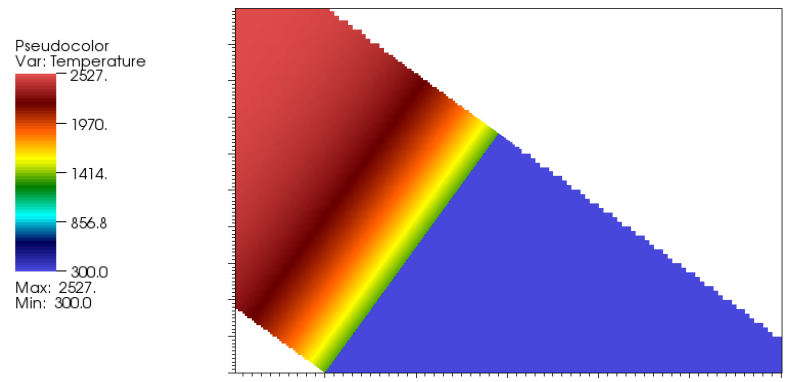

(a) Temperature

Figure 14: Temperature (K) pseudo-color plot for ZND initial condition with one-step chemistry.

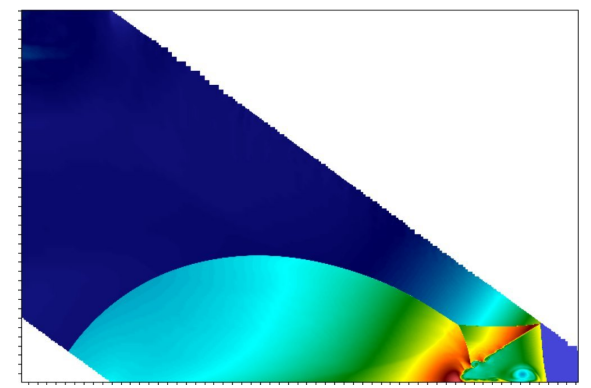

(a) Density

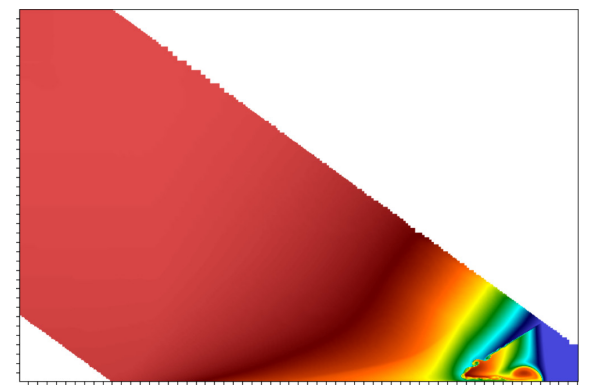

(b) Product Mass Fraction

Figure 15: Pseudo-color plots for a fully-resolved marginally stable detonation with one-step chemistry.

A simple expression that includes the inverse dependence on pressure was used for the mass diffusion

$$
D_{1}=D_{1 \text { ref }}\left(\frac{T}{T_{\text {ref }}}\right)^{\frac{3}{2}} \frac{T}{T_{\text {ref }}} \frac{p_{\text {atm }}}{p}, \quad D_{2}=D_{2 \text { ref }}\left(\frac{T}{T_{\text {ref }}}\right)^{\frac{3}{2}} \frac{T}{T_{\text {ref }}} \frac{p_{\text {atm }}}{p} .
$$

At the end of the reaction zone, the temperature is approximately $2500 \mathrm{~K}$ and the pressure is $\sim 1 \mathrm{~atm}$. This yields the following transport parameters for our one-step model: $T_{\text {ref }}=2500 \mathrm{~K}, \mu_{\text {ref }}=1.07 \cdot 10^{-4} \mathrm{Pas}, k_{\text {ref }}=0.148 \mathrm{~W} /(\mathrm{mK})$, $D_{1 \text { ref }}=5.5 \cdot 10^{-4} \mathrm{~m}^{2} / \mathrm{s}, D_{2 \text { ref }}=6.4 \cdot 10^{-4} \mathrm{~m}^{2} / \mathrm{s}$. Note that for calculating a Reynolds number we use $\mu_{\text {ref }}$ rather than $\mu_{\infty}$ as $\mu$ in this case is a function of temperature.

\subsubsection{One-step chemistry}

Results for the whole domain are presented in Figures 14-15(a). For this simulation, the ZND planar steady detonation wave solution was used as initial condition. Using an average density, $\rho \approx 0.60 \mathrm{~kg} / \mathrm{m}^{3}$, of the top and bottom portions of the shear layer directly behind the triple point, viscosity of $5.95 \cdot 10^{-5} \mathrm{~Pa}$ s, thermal conductivity of $0.847 \mathrm{~W} /(\mathrm{mK})$, a mass diffusivity of $1.57 \cdot 10^{-4} \mathrm{~m}^{2} / \mathrm{s}$ for the average temperature of $2000 \mathrm{~K}$, and a pressure of $2 \mathrm{~atm}$ the diffusive scales were estimated (see Table $8(\mathrm{~A})$ ). Observe that the viscous scale is the smallest, and mass diffusion and heat diffusion scale are approximately 1.5 and 10 times larger, respectively.

\subsubsection{Convergence results}

Using the nondimensional version of the equations and up to 8-levels of refinement, convergence of the method for the reactive DMR was verified as shown in Table 8 (B) to (D). For these simulations, the National Energy Research Scientific Computing Center machine "Carver" (quad-core Intel 5500 series) was used with 128 cores. Additionally, in order to increase the efficiency of the simulation and allow for higher resolution, a time-dependent coarsening region behind the DMR was utilized, as shown in Figure 16(b). Three-step RK3 integration was used with a CFL time step parameter of 0.99 in conjunction with the time-split integration for the chemistry. As expected, it was found that when the induction length is larger than the viscous scales (as is for realistic physical parameters), a fully resolved simulation is limited by the viscous length scale. Shown in Figure 16(c) is the use of the WENO scheme only at the strong shocks. At the highest resolution, the shock coming from the second triple point is not strong enough to activate 


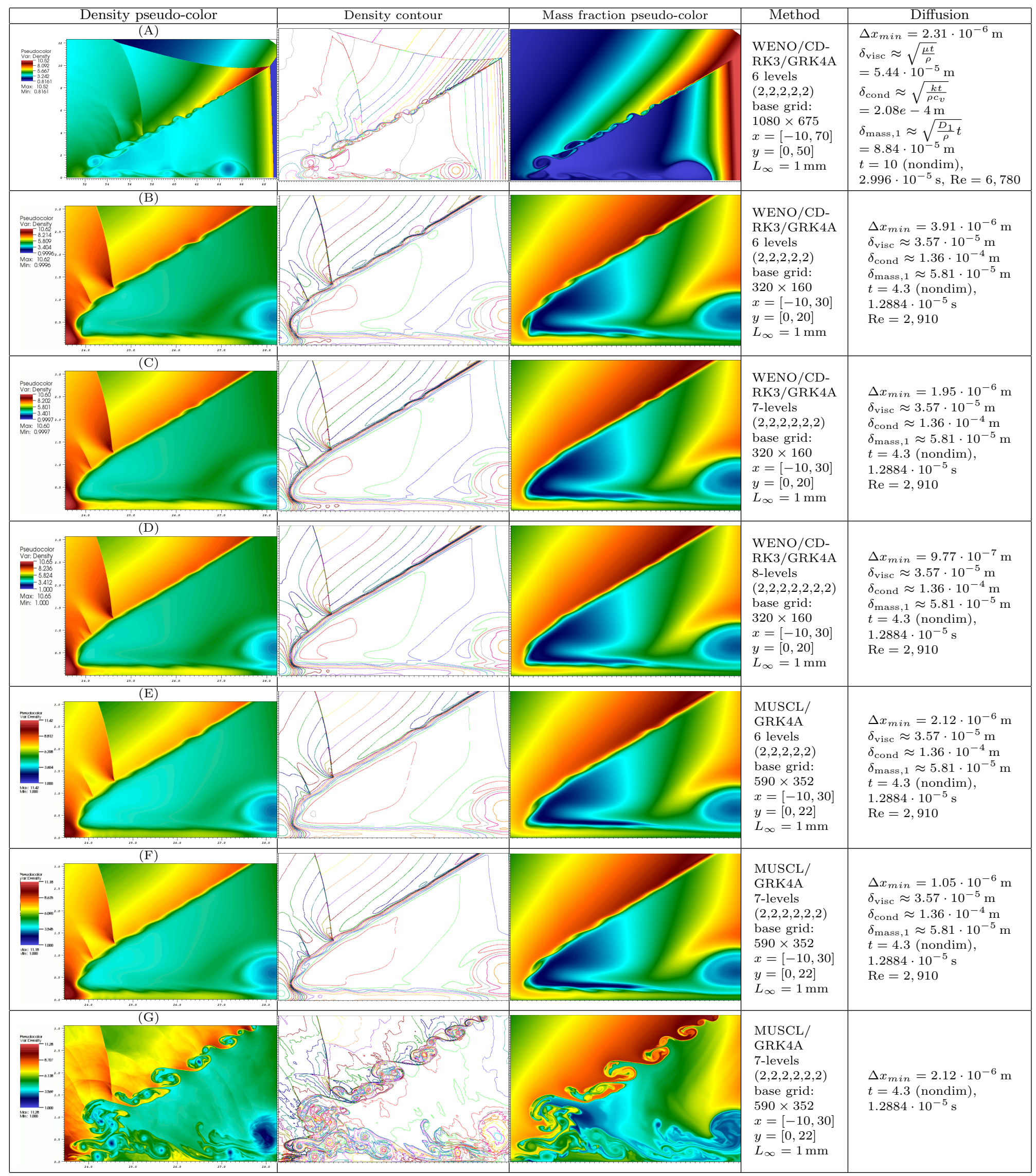

Table 8: Reactive diffusive double Mach reflection: density, density contour, and mass fraction pseudo-color plots of the DMR structure. Figure (A) demonstrates the long-term behavior and Figures (B) to (D) demonstrate the convergence of the WENO/CD method in resolving the viscous processes in the mixing layer, by showing a $2 \times$ increase in resolution for 3 simulations. The approximate viscous scale and minimum cell size give the required resolution for convergence of the viscous DMR problem. Figures (E) to (F) show the convergence of a standard MUSCL scheme for the same problem. Figure (G) shows the equivalent inviscid simulation. Note: For the electronic version, be sure to use the zoom tool in the PDF reader to study the details of the high resolution plots. 


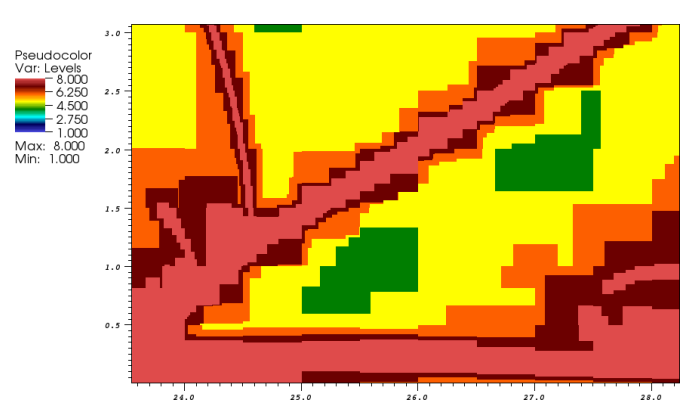

(a) Refinement levels

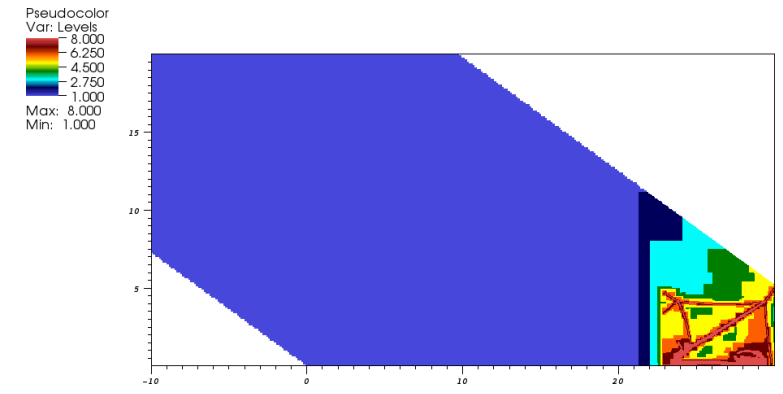

(b) Refinement levels

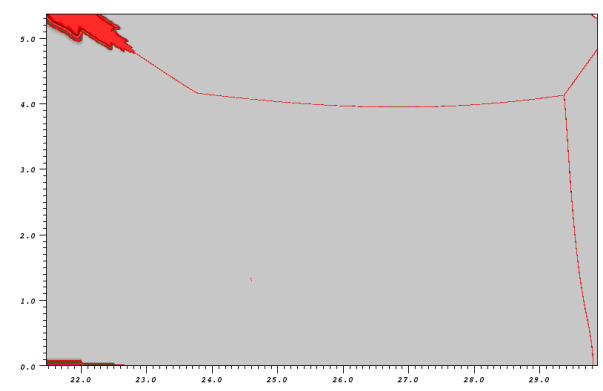

(c) WENO usage (red)

Figure 16: SAMR levels and WENO usage for the 8-level reactive DMR convergence test.

\begin{tabular}{|c|c|c|c|c|}
\hline Levels & $\begin{array}{l}\text { Density } \\
L_{1} \text {-error }\end{array}$ & rate & $\begin{array}{c}Y_{1} \\
L_{1} \text {-error }\end{array}$ & rate \\
\hline \multicolumn{5}{|l|}{$t=4.300$, domain: $[23.0,30.0] \times[0,5.0]$} \\
\hline 4 & 2.00432 & - & 0.216785 & - \\
\hline 5 & 1.36915 & 0.55 & 0.160973 & 0.43 \\
\hline 6 & 0.709029 & 0.95 & 0.0791281 & 1.03 \\
\hline 7 & 0.329581 & 1.11 & 0.0360734 & 1.13 \\
\hline \multicolumn{5}{|l|}{$\begin{array}{c}\mathrm{t}=4.608 \text { domain: }[23.0,30.0] \times[0,5.0] \\
(\text { Mach stem/incident shock has left the domain })\end{array}$} \\
\hline 4 & 1.83941 & - & 0.226811 & - \\
\hline 5 & 1.36174 & 0.44 & 0.170671 & 0.41 \\
\hline 6 & 0.810797 & 0.75 & 0.104466 & 0.71 \\
\hline 7 & 0.350756 & 1.21 & 0.0429677 & 1.28 \\
\hline
\end{tabular}

Table 9: $L_{1}$-error norms for the state variables using the 8-level case as the reference solution.

the discontinuity flag and the centered difference method captures the shock satisfactorily. At these resolutions, the weak shocks are nearly resolved, yet the strong shocks remains under-resolved.

Again, using the highest resolved 8-level case as a reference solution, $L_{1}$-error norms were calculated for the density and first species mass fraction to quantify the convergence of the reactive DMR problem. Quantitative results are given in Table 9; pseudo-color plots of the absolute value of the cell-wise difference between current and reference approximation are displayed in Table 10. As previously shown for the nonreactive case, the solution is converging and accurately resolved. The latter statement is further supported by the visualization of the local errors in Table 10, showing obvious visual convergence in the shear layer.

\subsubsection{Method comparison}

Using up to 7-levels refinement, the computation was repeated with the MUSCL method and second-order accurate finite differences for the diffusive terms. As shown in Table 8 (E) to (F), the overall flow field is similar, including the position of the shock waves, the jet, and the mixing layer. However, not surprisingly, is the observed result that the roll-up of the shear layer occurs later, which is surmised to be from the additional numerical viscosity (from the spatial solver and temporal integrator) and from the second-order errors from linear interpolation across the SAMR levels. Also for comparison, an inviscid solution is shown in Table $8(\mathrm{G})$. In this case, it is the numerical viscosity (which is a function of the resolution used) that dictates the roll-up of the mixing layer. This last case stresses the importance of including physical rather than numerical viscosity when simulating detonations as most detonation simulations us the 


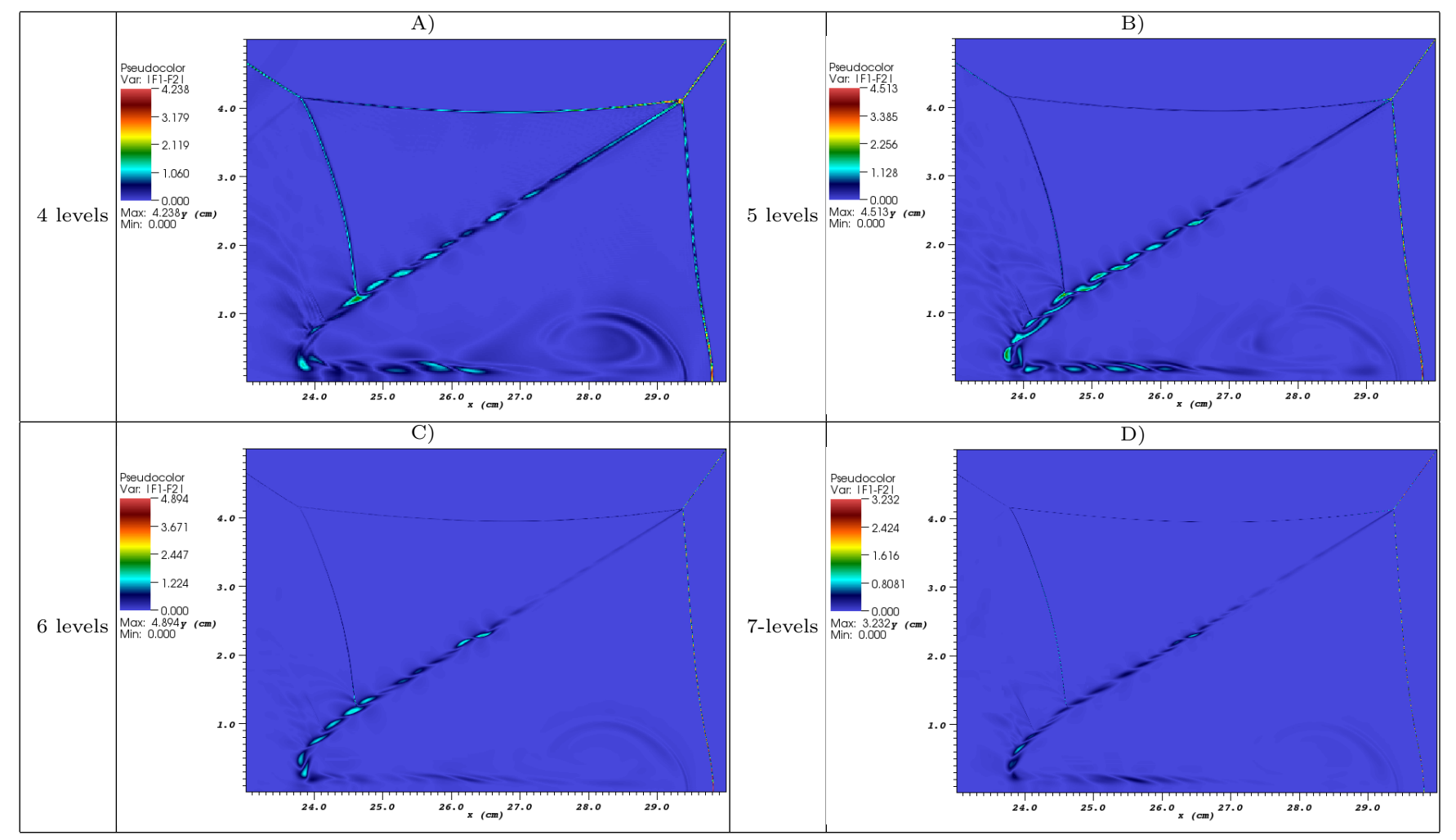

Table 10: Density errors calculated with a comparison to the highest resolved case (8-levels).

\begin{tabular}{|c|c|c|c|}
\hline Scheme & Levels & Wall time & CPU time \\
\hline MUSCL & 6 & $6.25 \cdot 10^{3} \mathrm{~s}=1.74 \mathrm{~h}$ & $55.6 \mathrm{~h}$ \\
MUSCL & 7 & $2.51 \cdot 10^{4} \mathrm{~s}=6.97 \mathrm{~h}$ & $233 \mathrm{~h}$ \\
MUSCL & 8 & $8.65 \cdot 10^{4} \mathrm{~s}=24.0 \mathrm{~h}$ & $769 \mathrm{~h}$ \\
\hline WENO/CD & 6 & $1.74 \cdot 10^{4} \mathrm{~s}=4.83 \mathrm{~h}$ & $155 \mathrm{~h}$ \\
WENO/CD & 7 & $5.28 \cdot 10^{4} \mathrm{~s}=14.7 \mathrm{~h}$ & $470 \mathrm{~h}$ \\
WENO/CD & 8 & $1.78 \cdot 10^{5} \mathrm{~s}=49.5 \mathrm{~h}$ & $1590 \mathrm{~h}$ \\
\hline
\end{tabular}

Table 11: Comparison of run times for the MUSCL and WENO/CD methods using one-step, two-component chemistry for the DMR problem. Intel 5400 series, 32 cores, $320 \times 160$ SAMR base grid, $x=(-10,30), y=(0,20)$, final time $t=4.91498476$

reactive Euler equations as an approximation.

Also, as shown in Table 11, is a comparison of the computational expense of the MUSCL and WENO/CD methods. Results are shown for 32 cores as actual wall time and total CPU hours. The WENO/CD method is approximately two times more expensive than MUSCL as the number of levels is increased while using the same base grid. Note that the wall times increase at a rate greater than linear as each level is added.

\section{3. $\mathrm{H}_{2}-\mathrm{O}_{2}-\mathrm{Ar}$ multicomponent chemistry}

Using our simplified two-component one-step chemistry model as a basis for comparison, the results were further extended to realistic, multicomponent, detailed chemistry. Adapted from Deiterding [19], detailed chemistry and transport of the diffusive DMR problem was modeled using a basic hydrogen-oxygen mechanism as extracted from the hydrocarbon mechanism by Westbrook [69]. This mechanism consists of 9 species $\mathrm{O}_{2}, \mathrm{H}_{2} \mathrm{O}, \mathrm{H}, \mathrm{O}, \mathrm{OH}, \mathrm{H}_{2}, \mathrm{HO}_{2}, \mathrm{H}_{2} \mathrm{O}_{2}, \mathrm{Ar}$ and 34 Arrhenius rate reactions. Due to the larger computational expense required by the additional species and chemistry terms, presently, the diffusive processes are two times less resolved than the previously presented twocomponent flow results. Because the reaction zone length scale is much larger than the diffusive scales, and we resolve the diffusive scales, the influence of the chemistry is completely resolved, as shown with the $\mathrm{OH}$ radical mass fraction field in Table 12. Note that the two-component one-step case was modeled to correspond to the detailed chemistry simulations in terms of the reactive and diffusive length scales. Also, except for the 8-level case, the smallest cell sizes are the same, and the refinement criteria are similar. Therefore, a comparison of the simulations supports that this detailed chemistry result, being similar to the one-step result, is also resolved in the 7-level case shown in Table 12. The expense of the highest resolved case was $140,000 \mathrm{~h}$ CPU, running 3 months continuously with 64 cores. An even further resolved simulation with 8-levels is currently unfeasible for us. Also note that, at the current resolution, a 


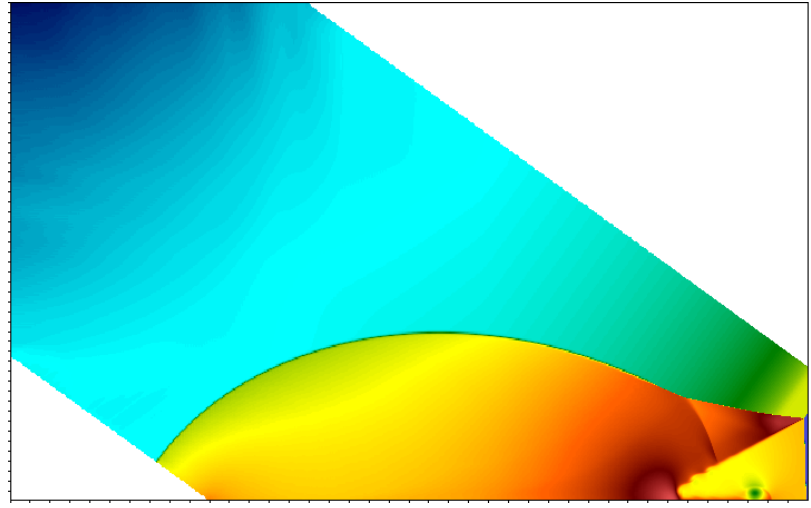

(a) A)

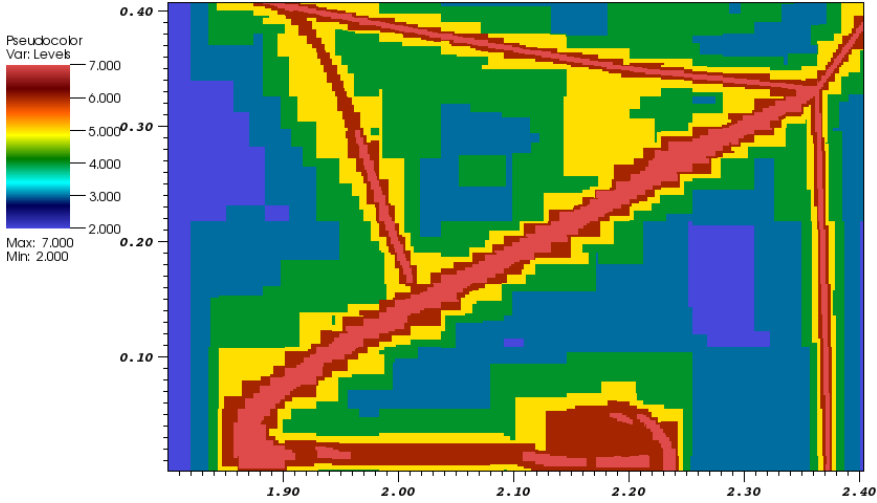

(b) B)

Figure 17: A) DMR density pseudo-color results with detailed chemistry and transport of an $\mathrm{H}_{2}-\mathrm{O}_{2}-\mathrm{Ar}$ detonation in a mixture of initial mole ratios of $2: 1: 7$ and at $T=300 \mathrm{~K}$ and $p=6,700 \mathrm{~Pa}$. The Chapman-Jouguet detonation speed is $1,627 \mathrm{~m} / \mathrm{s}$ and the induction length $0.01875 \mathrm{~m}$. Problem setup: $[-0.010,0.030] \mathrm{m} \times[0.0,0.022] \mathrm{m}, 7$ levels $(2,2,2,2,2,2), 590 \times 369$ base grid, $\Delta x_{\mathrm{min}}=1.06 \cdot 10^{-6} \mathrm{~m}$, $t=1.2829 \cdot 10^{-5} \mathrm{~s}$. B) Refinement levels of the DMR at a time of $t=1.05386 \cdot 10^{-5}$

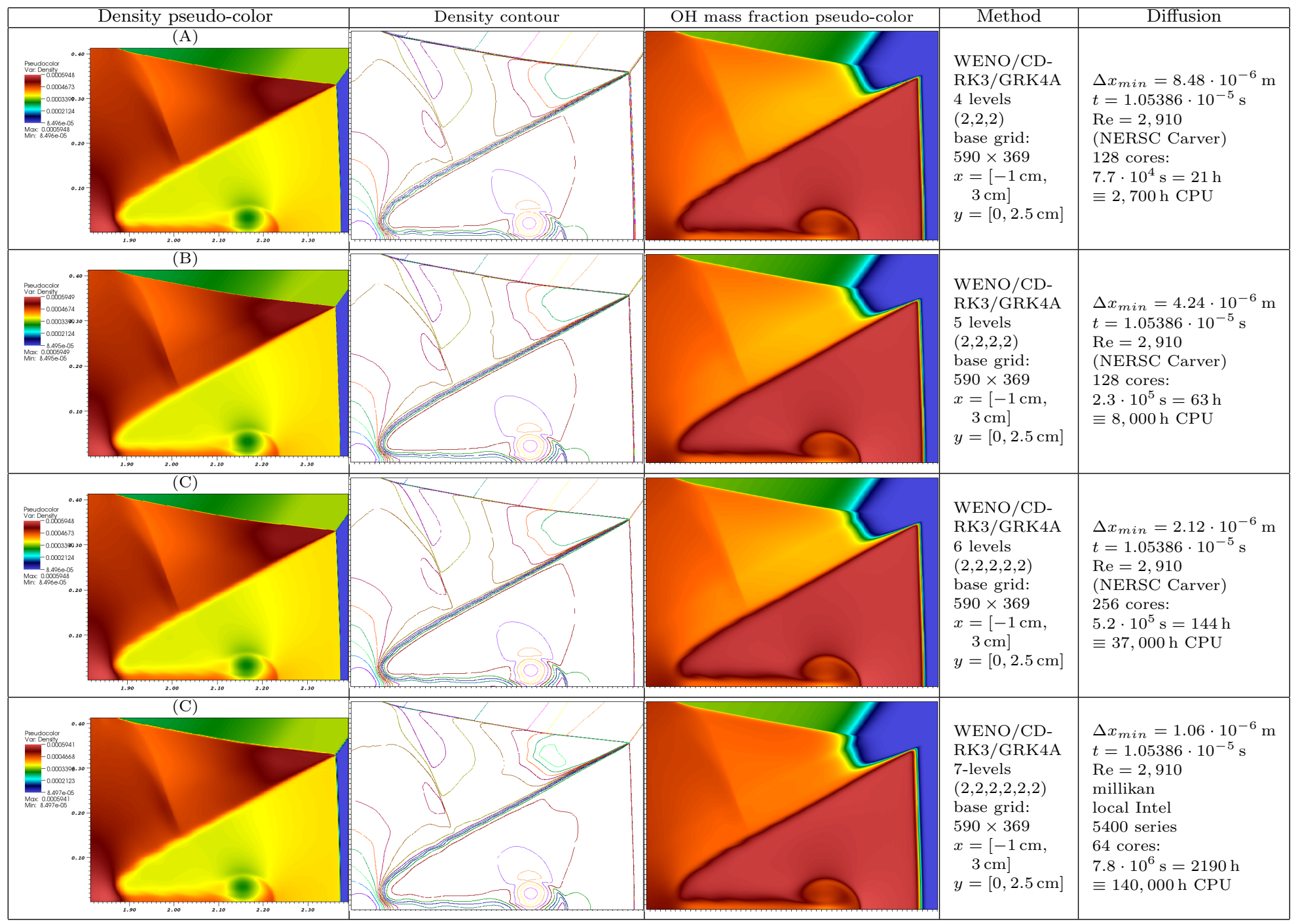

Table 12: Reactive diffusive double Mach reflection with detailed chemistry: density, density contour, and mass fraction pseudo-color plots of the DMR structure. 


\begin{tabular}{|c|c|c|c|c|c|c|}
\hline Levels & $\begin{array}{l}\text { density } \\
L_{1} \text {-error }\end{array}$ & rate & $\begin{array}{c}\text { total energy } \\
L_{1} \text {-error }\end{array}$ & rate & $\begin{array}{c}\mathrm{OH} \text { mass fraction } \\
L_{1} \text {-error }\end{array}$ & rate \\
\hline \multicolumn{7}{|l|}{ A) } \\
\hline 3 & $1.030 \mathrm{E}-6$ & - & $3.531 \mathrm{E}+4$ & - & $1.729 \mathrm{E}-4$ & - \\
\hline 4 & $7.445 \mathrm{e}-07$ & 0.468 & $2.270 \mathrm{E}+4$ & 0.637 & $1.589 \mathrm{E}-4$ & 0.122 \\
\hline 5 & $6.849 \mathrm{e}-07$ & 0.124 & $2.037+4$ & 0.047 & $1.575 \mathrm{E}-4$ & 0.013 \\
\hline 6 & $6.723 \mathrm{e}-07$ & 0.027 & $2.020 \mathrm{E}+4$ & 0.004 & $1.565 \mathrm{E}-4$ & 0.009 \\
\hline \multicolumn{7}{|l|}{ B) } \\
\hline 3 & $1.418 \mathrm{E}-6$ & - & $4.447 \mathrm{E}+4$ & - & $7.802 \mathrm{E}-5$ & - \\
\hline 4 & $1.306 \mathrm{E}-6$ & 0.119 & $4.141 \mathrm{E}+4$ & 0.103 & $6.899 \mathrm{E}-5$ & 0.177 \\
\hline 5 & $1.288 \mathrm{E}-6$ & 0.020 & $4.013 \mathrm{E}+4$ & 0.045 & $6.887 \mathrm{E}-5$ & 0.003 \\
\hline 6 & $1.276 \mathrm{E}-6$ & 0.013 & $3.991 \mathrm{E}+4$ & 0.008 & $6.778 \mathrm{E}-5$ & 0.023 \\
\hline
\end{tabular}

Table 13: Detailed chemistry convergence results: $L_{1}$-error norms for the state variables using the 7 -level case as the reference solution: A) final time: $t=1.05386 \cdot 10^{-5} \mathrm{~s}$, domain: $[1.8,2.4] \times[0,0.4] \mathrm{cm}$, corresponding to when the mixing layer starts to roll up. B) final time: $t=1.1455 \cdot 10^{-5} \mathrm{~s}$, domain: $[2.026,2.47] \times[0,0.32] \mathrm{cm}$

nondimensionalization was unnecessary to avoid underflow errors.

Results are presented for the whole domain in Figure 17 and for the area spanned by the DMR in Table 12 . Using the 7-level case as an "exact" solution, equivalent convergence rates were calculated and are shown Table 13 . The rates are significantly lower than for the previous configurations. Although the images in Table 12 seem to suggest convergence, the quantitative analysis and visualizations of the local error similarly as in Table 10 (not shown here) uncover that the computational resolution and refinement criterion were not yet sufficient. In the highest resolved detailed chemistry case, the thickness of the last refinement level around the shear layer is thinner than that used in the 7- and 8-level two-component one-step simulations. Our rates are calculated assuming that all levels are simultaneously made two times finer, where in practice just the finest level is refined. Therefore, the magnitude of the rates is dependent on the refinement criterion, specifically the efficency parameter and error tolerances. The conclusive quantitative convergence results of the two-species case support the validity of the multicomponent results and also demonstrate some of the differences in a solution when choosing to model detailed chemistry. There are now multiple reactions scales, one of which, $\mathrm{OH}$, is shown in Table 12. These simulations were run only until the onset of instability in the shear layers and a careful verification study was used to quantify our confidence in the validity of the results. Note that comparisons past the onset of the initial instability are problematic as the flow in the shear layer leaves the laminar regime.

The parameters selected for this case correspond to a regular detonation structure [64] with a nearly laminar reaction zone and periodically-spaced transverse waves. This corresponds to a weakly unstable mixture characteristic of low values of the effective activation energy [57] with a relative long energy release region compared to the induction zone length. The reaction zone fluctuations are quasi-steady [64] in weakly unstable waves, and the shear layers associated with the triple-points are primarily downstream of the induction zone. However, due to the subsonic (relative to the shock front) nature of the flow field, the dynamics of the mixing layers can influence the overall structure. In addition, there is clear experimental evidence [63] of jetting of relatively unreacted fluid into the reacted region in the weakly unstable case.

In the present simulations, the results show that there is a clear difference between simulations with and without diffusive transport in the region of the shear layers and jetting. As expected, the presence of diffusion dramatically affects the presence of the large-scale structures in the shear layer, features that are very prominent [61, 48] in inviscid simulations of detonations. However, since we have not yet performed simulations of self-sustaining propagating detonations, it is unclear how diffusion will influence the qualitative and quantitative conclusions drawn from inviscid simulations. Of course, the role of diffusion will be strongly dependent on the characteristic Reynolds number associated with the shear flow. In the present case, a characteristic value of $\operatorname{Re} \sim 3000$ is representative of fuel-oxygen detonations at low pressure, similar to those examined in Radulescu et al. [61]. Larger values are possible in fuel-air mixtures at high pressures. Extensive simulations of detonation structure with models of diffusive processes have been carried out in two and three space dimensions by the group at the Naval Research Laboratory, see for example, Kessler et al. [41]. They simulated these flows using the reactive Navier-Stokes equations and one-step models with diffusive transport of a single species. The emphasis of these studies has primarily been on deflagration to detonation transition and comparisons of inviscid and viscous propagating detonation simulations are not presented.

The present study is most relevant to the simulation of irregular detonations which are characterized by a turbulent reaction zone and a large range of spatial scales both in the spacing of transverse waves, the associated shear layers and reaction fronts $[3,60]$. Motivated by the experimental observations, preliminary $[2,52]$ studies of diffusive reaction along shear layers have been carried out by previous authors. In particular, Massa et al. [52] carried out a two-dimensional simulation of the shear layer behind expected detonation triple points. Note that this is unlike our simulations in that it excludes all shock waves and symmetry boundary conditions which create the bottom jet. They investigated the role 
of vortical structures associated with Kelvin-Helmholtz $(\mathrm{KH})$ instability in the formation of localized ignition using detailed chemical kinetics and transport. Their analysis indicates that the diffusive processes and KH instability play no role in ignition for low activation energy mixtures and have a modest role in ignition events for high activation energy mixtures. However, the model problem that Massa et al. examined was highly idealized and it is difficult to generalize the results to the structure of propagating detonations.

The present simulations are encouraging and demonstrate the feasibility of applying the computational method to the investigation of the mechanisms involved in the diffusive processes of irregular detonations. Ultimately, the goal is to study highly unstable mixtures for which major effects along the shear layers can be expected; e.g., unreacted pockets transported downstream or reaction behind highly fluctuating shock fronts. The goal in the foreseeable future would be to apply these methods to two-dimensional simulations of irregular detonation problems with a reduced model of chemistry and transport. Even with the ongoing advances in computational capability, it seems unlikely that simulations will be possible in the near term with realistic reaction mechanisms for hydrocarbon fuels in three-dimensional flows.

\section{Concluding remarks}

The present convergence results for the non-reactive and reactive DMR simulations support the case that, with our implementation and detailed simulations, diffusive processes within chemically reacting zones can be resolved. The advances that have allowed this are as follows: First, careful use of a hybrid method, where WENO is activated only at strong shock waves (using an approximate Riemann-problem based shock detection), allowed the sixth-order accurate centered difference stencils to be uniformly active on shear layers and surrounding regions. Additionally, away from the shocks, fifth-order accurate prolongation/restriction operators were utilized on the fine/coarse mesh boundaries and overlaps while first-order operators were used near the strong shocks in order to provide stability. Second, the detailed inclusion of a reliable diffusive stability condition for the explicit RK4 and RK3 integration allowed to take maximum time steps. Third, appropriate nondimensionalization using physically relevant parameters was found to be necessary for the elimination of underflow errors. Finally, a study of mesh refinement indicated that a minimum coverage, at the finest refinement level, was required in the region surrounding the shear layers in order to obtain convergence. The results produced using the present implementation, together with advancements in resolving capability and accuracy, demonstrate possible future directions for converged SAMR simulations.

\section{Acknowledgements}

Jack Ziegler is supported by the Department of Energy Computational Science Graduate Fellowship program (DOE CSGF). This research used resources of the National Energy Research Scientific Computing (NERSC) Center. D. Pullin and R. Deiterding were partially supported by the Department of Energy Advanced Scientific and Computing (ASC) program under subcontract B341492 of DOE contract W-7405-ENG-48.

\section{A. Nondimensionalization}

A nondimensionalization or scaling of the fluid dynamic equations is important at the highest resolutions. Without a scaling the equations are ill-conditioned for a large pressure and small time step (short diffusive and reactive time scale). When simulating diffusive flow with realistic physical parameters and using fine grids in order to show convergence, the time steps become so small that round-off errors and lack of precision pollute the results. Nondimensionalization mitigates this problem. The non-reactive results were for a shock traveling into standard atmospheric conditions ( $T=300 \mathrm{~K}$ and $p=1.01325 \cdot 10^{5} \mathrm{~Pa}$ ); however, the preliminary detonation simulations were for a shock propagating into a lower pressure of $6,700 \mathrm{~Pa}$, for which a nondimensionalization was unnecessary.

\section{A.1. Non-reactive Navier-Stokes equations}

In the non-reactive case, using the length and the time scaling $x=L_{\infty} x^{*}, y=L_{\infty} y^{*}$ and $t=\frac{L_{\infty}}{a_{\infty}} t^{*}$, respectively, the primitive variables are nondimensionalized as

$$
u=a_{\infty} u^{*}, \quad v=a_{\infty} v^{*}, \quad \rho=\rho_{\infty} \rho^{*}, \quad p=\rho_{\infty} a_{\infty}^{2} p^{*}, \quad T=\frac{a_{\infty}^{2}}{c_{p_{\infty}}} T^{*}
$$

with the ${ }^{*}$ as nondimensional. The normalized caloric equation and ideal gas law for a single polytropic gas then read

$$
c_{p}(T)=c_{p_{\infty}} c_{p}^{*}\left(T^{*}\right), \quad R=c_{p_{\infty}} R^{*}, \quad p=\rho R T, \quad\left(\rho_{\infty} a_{\infty}^{2} p^{*}\right)=\left(\rho_{\infty} \rho^{*}\right)\left(c_{p_{\infty}} R^{*}\right)\left(\frac{a_{\infty}^{2}}{c_{p_{\infty}}} T^{*}\right) .
$$


The validity of this nondimensionalization can be verified easily by substituting these variables into the Navier-Stokes equations and canceling the factors to derive the results below. The nondimensional non-reactive compressible NavierStokes equations in two space dimensions are

$$
\begin{aligned}
\frac{\partial \rho^{*}}{\partial t^{*}}+\frac{\partial \rho^{*} u^{*}}{\partial x^{*}}+\frac{\partial \rho^{*} v^{*}}{\partial y^{*}} & =0 \\
\frac{\partial \rho^{*} u^{*}}{\partial t^{*}}+\frac{\partial \rho^{*} u^{* 2}+p^{*}}{\partial x^{*}}+\frac{\partial \rho^{*} u^{*} v^{*}}{\partial y^{*}} & =\frac{\partial \tau_{x x}^{*}}{\partial x^{*}}+\frac{\partial \tau_{x y}^{*}}{\partial y^{*}}, \\
\frac{\partial \rho^{*} v^{*}}{\partial t^{*}}+\frac{\partial \rho^{*} u^{*} v^{*}}{\partial x^{*}}+\frac{\partial \rho^{*} v^{* 2}+p}{\partial y^{*}} & =\frac{\partial \tau_{x y}^{*}}{\partial x^{*}}+\frac{\partial \tau_{y y}^{*}}{\partial y^{*}}, \\
\frac{\partial \rho^{*} e_{t}^{*}}{\partial t^{*}}+\frac{\partial \rho^{*} u^{*}\left(e_{t}^{*}+p^{*}\right)}{\partial x^{*}}+\frac{\partial \rho v^{*}\left(e_{t}^{*}+p^{*}\right)}{\partial y^{*}} & =\frac{\partial\left(u^{*} \tau_{x x}^{*}+v^{*} \tau_{x y}^{*}\right)}{\partial x^{*}}+\frac{\partial\left(u^{*} \tau_{y x}^{*}+v^{*} \tau_{y y}^{*}\right)}{\partial y^{*}}+\frac{\partial q_{x}^{*}}{\partial x^{*}}+\frac{\partial q_{y}^{*}}{\partial x^{*}},
\end{aligned}
$$

where the stresses read

$$
\tau_{x x}^{*}=\mu^{*}\left(T^{*}\right)\left(2 \frac{\partial u^{*}}{\partial x^{*}}-\frac{2}{3}\left(\frac{\partial u^{*}}{\partial x^{*}}+\frac{\partial v^{*}}{\partial y^{*}}\right)\right), \tau_{x y}=\mu^{*}\left(T^{*}\right)\left(\frac{\partial u^{*}}{\partial y^{*}}+\frac{\partial v^{*}}{\partial x^{*}}\right), \tau_{y y}=\mu^{*}\left(T^{*}\right)\left(2 \frac{\partial v^{*}}{\partial y^{*}}-\frac{2}{3}\left(\frac{\partial u^{*}}{\partial x^{*}}+\frac{\partial v^{*}}{\partial y^{*}}\right)\right)
$$

and the heat transfer terms are

$$
q_{x}^{*}=k^{*}\left(T^{*}\right) \frac{\partial T^{*}}{\partial x^{*}}, \quad q_{y}^{*}=k^{*}\left(T^{*}\right) \frac{\partial T^{*}}{\partial y^{*}}
$$

with normalized mixture viscosity and normalized thermal conductivity

$$
\mu=a_{\infty} L_{\infty} \rho_{\infty} \mu^{*}\left(T^{*}\right), \quad k=\rho_{\infty} a_{\infty} L_{\infty} c_{p_{\infty}} k^{*}\left(T^{*}\right)
$$

\section{A.2. Reactive Navier-Stokes equations for thermally perfect mixtures}

For the case of $N$ thermally perfect species, the normalization of the total and partial densities is $\rho_{\infty}=\sum_{i=1}^{N} \rho_{i \infty}$, $\rho_{i}=\rho_{\infty} \rho_{i}^{*}$ and the normalized caloric equations (50) take the form

$$
c_{p_{i}}(T)=c_{p_{\infty}} c_{p_{i}}^{*}\left(T^{*}\right), h_{i}(T)=a_{\infty}^{2} h_{i}^{*}, R_{i}=c_{p_{\infty}} R_{i}^{*}
$$

The normalized mass diffusivities read $D_{i}(T)=a_{\infty} L_{\infty} D_{i}^{*}\left(T^{*}\right)$ and, in case of chemical reaction, the normalized Arrhenius parameters are

$$
E_{i}=a_{\infty}^{2} E_{i}^{*}, \quad A_{i}=\frac{a_{\infty}}{L_{\infty}} A_{i}^{*}
$$

A.2.1. Navier-Stokes equations for two callorically perfect gases with one-step reaction

If only two calorically perfect gases with identical adiabatic coefficient, $\gamma$, are considered, (58) reduces to

$$
c_{p}=\frac{\gamma R}{\gamma-1}, \quad c_{p}=c_{p} c_{p}^{*}, \quad c_{p} *=1, \quad h_{1}=q_{0}+c_{p} T, \quad h_{2}=c_{p} T, \quad q_{0}=a_{\infty}^{2} q_{0}^{*}, \quad R=c_{p} R^{*}
$$

where the normalized temperature is now given as $T=\frac{a_{\infty}^{2}}{c_{p}} T^{*}$. With the single reaction

$$
\frac{d \omega_{1}}{d t}=\rho_{1} A \exp \left(\frac{E_{A}}{R_{u} T}\right)=\rho_{1} A \exp \left(\frac{E}{R T}\right)
$$

the normalized Arrhenius parameters of (59) read

$$
E=\frac{E_{A}}{W}=E_{A} \frac{R}{R_{u}}, \quad E=a_{\infty}^{2} E^{*}, \quad A=\frac{a_{\infty}}{L_{\infty}} A^{*} .
$$

\section{B. Stability criterion}

Special care must be taken when defining a stable, explicit, time-step criterion for mixed type nonlinear PDEs. By considering a straightforward explicit finite difference scheme for the closely related multidimensional but scalar advection-diffusion equation with constant coefficients

$$
\frac{\partial \phi}{\partial t}+u \frac{\partial \phi}{\partial x}+v \frac{\partial \phi}{\partial y}=K_{x} \frac{\partial^{2} \phi}{\partial x^{2}}+K_{y} \frac{\partial^{2} \phi}{\partial y^{2}}
$$


an approximate criterion for explicit methods for the Navier-Stokes equations is constructed. The stability criterion for a finite difference method for (63) is found by von Neumann stability analysis. Then using this results as an analogue, a combined stability criterion for explicit schemes for the Navier-Stokes equations is derived. The parameters resulting from this analysis are

$$
\alpha_{x}=\frac{2 K_{x} \Delta t}{\Delta x^{2}}, \quad C_{x}=\frac{u \Delta t}{\Delta x}, \quad \alpha_{y}=\frac{2 K_{y} \Delta t}{\Delta y^{2}}, \quad C_{y}=\frac{v \Delta t}{\Delta y}
$$

where $\alpha_{x, y}$ and $C_{x, y}$ are the diffusion parameters and the Courant numbers for (63), respectively. The stability criterion for a hybrid finite difference method with forward in time, upwinded advection (backward space) and centered diffusion (FT-BS/CS) scheme in two space dimensions is simply [33]

$$
\alpha_{x}+C_{x}+\alpha_{y}+C_{y} \leq 1
$$

\section{B.1. Non-reactive explicit stability criterion}

To determine the stability limit for the used schemes for the non-reactive single-component Navier-Stokes equations one must look at each of the four conservation equations and determine the corresponding Courant numbers and diffusion parameters. For all four equations, the Courant number is the typical CFL number as for the inviscid Euler equations, cf. Section 3.4. The continuity equation for the density has only the Courant number stability criterion. The two momentum equations have the Courant number and a diffusion parameter, where the equivalent $K_{x}, K_{y}$ values for an advection-diffusion equation analogue are both $\frac{4}{3} \frac{\mu}{\rho}$. In the equation of the total energy density, the dissipative term from the viscosity does not directly affect the stability of $e_{t}$ and therefore the diffusion parameter comes from the heat conduction term, where now the analogous condition is $K_{x, y} \equiv \frac{k}{c_{v} \rho}$, with $k$ denoting the heat diffusivity and $c_{v}$ the specific heat at constant volume, $c_{v}=c_{p}-R$. Then, one splits the convective and diffusive parts, and the maximum time steps are constrained by different convective and diffusive scales. The maximum time step is limited by the combination of the convective CFL number with the diffusion parameters from viscosity and conductivity in the $x$ - and $y$-direction. For example, just from the $x$-direction, there is

$$
\nu_{\mathrm{conv}, x}=\frac{\Delta t}{\Delta x} \max |\lambda|,
$$

and the diffusive "CFL analogue" parameters are

$$
\nu_{\mathrm{diff}, \mathrm{visc}, x}=\frac{8 \mu \Delta t}{3 \rho(\Delta x)^{2}}, \quad \nu_{\mathrm{diff}, \mathrm{cond}, x}=\frac{2 k \Delta t}{c_{v} \rho(\Delta x)^{2}} .
$$

The complete stability condition encompassing convection, viscous diffusion, and heat diffusion is

$$
\nu=\max \left(\quad \nu_{\text {conv }}+\nu_{\text {diff, }, \text { isc }}, \quad \nu_{\text {conv }}+\nu_{\text {diff, cond }}\right) \leq 1,
$$

where in the two-dimensional case those quantities take the forms

$$
\nu_{\mathrm{conv}}=\frac{\Delta t}{\Delta x} \max (|u|+a)+\frac{\Delta t}{\Delta y} \max (|v|+a), \nu_{\mathrm{diff}, \mathrm{visc}}=\frac{8 \mu \Delta t}{3 \rho(\Delta x)^{2}}+\frac{8 \mu \Delta t}{3 \rho(\Delta y)^{2}}, \nu_{\mathrm{diff}, \mathrm{cond}}=\frac{2 k \Delta t}{c_{v} \rho(\Delta x)^{2}}+\frac{2 k \Delta t}{c_{v} \rho(\Delta y)^{2}} .
$$

Note that in this case the stability limit is computed from the contribution of the CFL numbers for all dimensions rather than taking the maximum of each dimension separately. The viscous and heat conduction scales are separated as they affect the stability of the momentum and energy equations separately. One must also note that in the case of using the $\operatorname{SSPRK}(10,4)$ scheme, the complete stability criterion is for $\nu \leq 6$, rather than $\nu \leq 1$, cf. Section 3.4. This criterion is for the total $\Delta t$, after all sub-steps have been taken. If one considers each sub-step separately, then on average the CFL number is for $\nu_{a v g} \leq 0.6$.

\section{B.2. Reactive multicomponent explicit stability criterion}

The stability of the two-dimensional multicomponent, reactive Navier-Stokes equations is found in the same fashion as for the non-reactive equations. The difference is now that the mass-averaged density, viscosity, and thermal conductivity are used for the convective, viscous, and thermal criterion. However, the mass diffusion of each species must be considered separately. In this case, the explicit stability condition is

$$
\nu=\max \left(\quad \nu_{\text {conv }}+\nu_{\text {diff }, \text { visc }}, \quad \nu_{\text {conv }}+\nu_{\text {diff }, \text { cond }}, \quad \nu_{\text {conv }}+\nu_{\text {diff }, \text { mass }}\right) \leq 1,
$$

with $\nu_{\text {diff, mass }}=\frac{D_{i}}{(\Delta x)^{2}}+\frac{D_{i}}{(\Delta y)^{2}}$, where $D_{i}$ is the mass diffusion parameter of the $i$-th species, cf. Section 2 . 


\section{Eigendecomposition}

The eigenvalues and eigenvectors of the thermally perfect multicomponent Euler equations can be derived in a straightforward algebraic computation, cf. [19]. For the state vector of conserved variables $\mathbf{q}=\left(\rho u, \rho v, \rho e_{t}, \rho Y_{1}, . ., \rho Y_{N}\right)^{T}$ the inviscid flux in the $x$-direction, for instance, satisfies $\mathbf{f}^{\text {conv }}=\mathbf{A}(\mathbf{q}) \mathbf{q}$, with

$$
\mathbf{A}(\mathbf{q})=\left[\begin{array}{ccccccc}
(3-\gamma) u & -\bar{\gamma} v & \bar{\gamma} & \phi_{1}-u^{2} & & \ldots & \phi_{N}-u^{2} \\
v & u & 0 & -u v & & \ldots & -u v \\
H-\bar{\gamma} u^{2} & -\bar{\gamma} u v & \gamma u & u\left(\phi_{1}-H\right) & & \ldots & u\left(\phi_{N}-H\right) \\
Y_{1} & 0 & 0 & u\left(1-Y_{1}\right) & -u Y_{1} & \ldots & -u Y_{1} \\
& & & -u Y_{2} & u\left(1-Y_{2}\right) & & \vdots \\
\vdots & \vdots & \vdots & \vdots & & \ddots & -u Y_{N-1} \\
Y_{N} & 0 & 0 & -u Y_{N} & \ldots & -u Y_{N} & u\left(1-Y_{N}\right)
\end{array}\right]
$$

denoting the corresponding Jacobian. For the set of eigenvalues $\lambda_{k}=\{u-a, u, \ldots, u, u+a\}$, the matrix of right eigenvectors, defined by $\left(\mathbf{A}-\lambda_{k} \mathbf{I}\right) \mathbf{r}_{k}=0$, reads

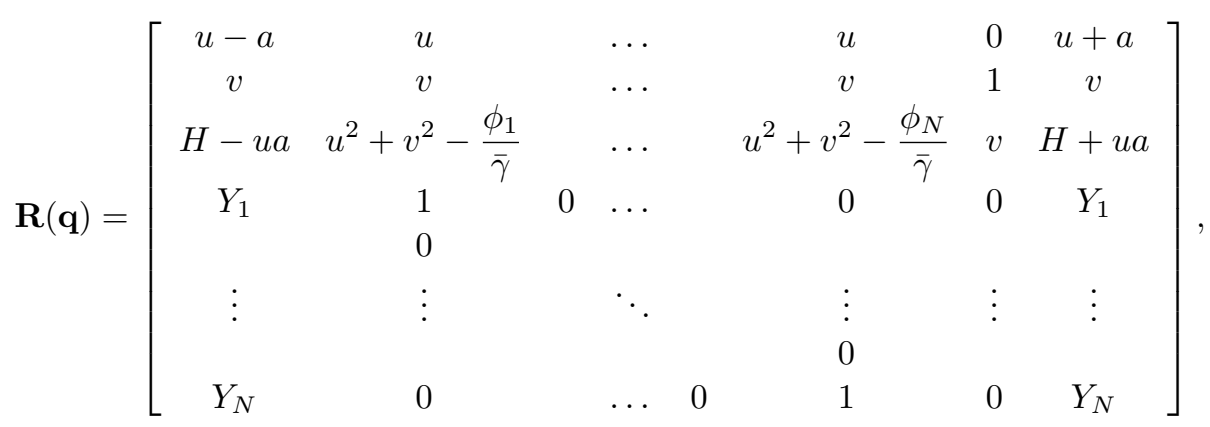

where $\phi_{i}=\frac{\partial p}{\partial \rho_{i}}$, the partial derivative of the pressure with respect to the $i$-th species' density, is given by

$$
\phi_{i}=\bar{\gamma}\left(\frac{u^{2}+v^{2}}{2}-h_{i}\right)+\gamma R_{i} T
$$

with $\bar{\gamma}=\gamma-1$ and the total specific enthalpy $H=h+\frac{u^{2}+v^{2}}{2}$.

\section{References}

[1] N. A. Adams and K. Sharif. A high-resolution hybrid compact-ENO scheme for shock-turbulence interaction problems. J. Comput. Physics, 127:27-51, 1996.

[2] M. Arienti and J. E. Shepherd. The role of diffusion in irregular detonations. In The 4th Joint Meeting of the US Sections of the Combustion Institute, Philadelphia, PA, March 20-23., 2005.

[3] J. M. Austin, F. Pintgen, and J. E. Shepherd. Reaction zones in highly unstable detonations. In Proc. Combust. Inst. 30, pages $1849-1857,2005$.

[4] D. S. Balsara and C. W. Shu. Monotonicity preserving weighted essentially non-oscillatory schemes with increasingly high order of accuracy. J. Comput. Physics, 160:405-452, 2000.

[5] G. Ben-Dor. A reconsideration of the three-shock theory for a pseudo-steady Mach reflection. J. Fluid Mechanics, 121:467-484, 1987.

[6] M. Berger and P. Colella. Local adaptive mesh refinement for shock hydrodynamics. J. Comput. Phys., 82:64-84, 1988.

[7] M. Berger and J. Oliger. Adaptive mesh refinement for hyperbolic partial differential equations. J. Comput. Phys., 53:484-512, 1984.

[8] R. Borges, M. Carmona, B Costa, and W. S. Don. An improved weighted essentially non-oscillatory scheme for hyperbolic conservation laws. J. Comput. Physics, 227:3191-3211, 2008.

[9] S. Browne, Z. Liang, R. Deiterding, and J. E. Shepherd. Detonation front structure and the competition for radicals. Proc. of the Combustion Institute, 31(2):2445-2453, 2007.

[10] J. Chao, A. Haselbacher, and S. Balachandar. A massively parallel multi-block hybrid compactWENO scheme for compressible flows. J. Comput. Physics, 228:7473-7491, 2009.

[11] P. Colella, M. Dorr, J. Hittinger, D. F. Martin, and P. McCorquodale. High-order finite-volume adaptive methods on locally rectangular grids. In J. Phys.: Conf. Ser., volume 180 012010, 2009.

[12] B. Costa and W. S. Don. High order hybrid central-WENO finite difference scheme for conservation laws. J. Comput. and App. Math., 204:209-218, 2007.

[13] M. S. Day, J. B. Bell, R. K. Cheng, S. Tachibana, V. E. Beckner, and M. J. Lijewski. Cellular burning in lean premixed turbulent hydrogen-air flames: coupling experimental and computational analysis at the laboratory scale. In SciDAC 2009, J. of Physics: Conference Series, San Diego, California, July 2009.

[14] M. De-Kang. Towards front-tracking based on conservation in two space dimensions II, tracking discontinuities in capturing fashion. J. Comput. Physics, 226:1550-1588, 2007. 
[15] R. Deiterding. Construction and application of an AMR algorithm for distributed memory computers. In T. Plewa, T. Linde, and V. G. Weirs, editors, Adaptive Mesh Refinement - Theory and Applications, volume 41 of Lecture Notes in Computational Science and Engineering, pages 361-372. Springer, 2005.

[16] R. Deiterding. Detonation structure simulation with AMROC. In L. T. Yang, editor, High Performance Computing and Communications 2005, volume 3726 of Lecture Notes in Computer Science, pages 916-927. Springer, 2005.

[17] R. Deiterding. A parallel adaptive method for simulating shock-induced combustion with detailed chemical kinetics in complex domains. Computers \& Structures, 87:769-783, 2009

[18] R. Deiterding. Parallel adaptive simulation of weak and strong detonation transverse-wave detonation structures in $\mathrm{h}_{2}-\mathrm{o}_{2}$ detonations. In R. Biswas, editor, Parallel CFD 2009: 21st Int. Conf. on Parallel Computational Fluid Dynamics, pages 519-534. DEStech Publications, 2010.

[19] R. Deiterding. Parallel Adaptive Simulation of Multi-dimensional Detonation Structures. PhD thesis, Brandenburgische Technische Universität Cottbus, 2003.

[20] R. Deiterding and G. Bader. High-resolution simulation of detonations with detailed chemistry. In G. Warnecke, editor, Analysis and Numerics for Conservation Laws, pages 69-91. Springer, 2005.

[21] R. Deiterding, R. Radovitzki, S. P. Mauch, F. Cirak, D. J. Hill, C. Pantano, J. C. Cummings, and D. I. Meiron. Virtual Test Facility: A virtual shock physics facility for simulating the dynamic response of materials. Available at http://www.cacr.caltech.edu/asc.

[22] R. Deiterding, R. Radovitzky, S. P. Mauch, L. Noels, J. C. Cummings, and D. I. Meiron. A virtual test facility for the efficient simulation of solid materials under high energy shock-wave loading. Engineering with Computers, 22(3-4):325-347, 2006.

[23] R. Deiterding, F. Cirak, S. P. Mauch, and D. I. Meiron. A virtual test facility for simulating detonation- and shock-induced deformation and fracture of thin flexible shells. Int. J. Multiscale Computational Engineering, 5(1):47-63, 2007.

[24] C. A. Eckett, J. J. Quirk, and J. E. Shepherd. The role of unsteadiness in direct initiation of gaseous detonation. J. Fliud Mech., 441: $142-183,2000$.

[25] R. P. Fedkiw, B. Merriman, and S. Osher. High accuracy numerical methods for thermally perfect gas flows with chemistry. J. Comput. Phys., 132:175-190, 1997

[26] S. Gottlieb and C.W. Shu. Total variation diminishing runge-kutta schemes. Combustion and Flame, 67:73-85, 1998.

[27] S. Gottlieb, D. I. Ketcheson, and C. W. Shu. High order strong stability preserving time discretizations. J. of Scientific Computing, $38(3): 251,2009$.

[28] J. A. Greenough and W. J. Rider. A quantitative comparison of numerical methods for the compressible Euler equations: fifth-order WENO and piecewise-linear Godunov. J. Comput. Phys., 196:259281, 2004.

[29] B. Gustafsson, H.-O. Kreiss, and J. Oliger. Time Dependent Problems and Difference Methods. John Wiley and Sons, 1995.

[30] A. K. Henrick, T. D. Aslam, and J. M. Powers. Mapped weighted essentially non-oscillatory schemes: Achieving optimal order near critical points. J. Comput. Physics, 207:542-567, 2005.

[31] A. K. Henrick, T. D. Aslam, and J. M. Powers. Simulations of pulsating one-dimensional detonations with true fifth order accuracy. J. Comput. Physics, 213:311-329, 2006.

[32] D. J. Hill and D. I. Pullin. Hybrid tuned center-difference-WENO method for large eddy simulations in the presence of strong shocks. J. Comput. Phys., 194(2):435-450, 2004.

[33] A. C. Hindmarsh and P. M. Gresho. The stability of explicit Euler time-integration for certain finite difference approximations of the multi-dimensional advection-diffusion equation. Inter. J. for Num. Meth. in Fluids, 4:853-897, 1984.

[34] A. E. Honein and P. Moin. Higher entropy conservation and numerical stability of compressible turbulence simulations. J. Comput. Phys., 201(2):531, 2004.

[35] P. Hwang, R. P. Fedkiw, B. Merriman, T. D. Aslam, A. R. Karagozian, and S. J. Osher. Numerical resolution of pulsating detonation waves. Appl. Numer. Math., 3:217-240, 2000.

[36] E. I. Vas Ilev, G. Ben-Dor, T. Elperin, and L. F. Henderson. The wall-jetting effect in Mach reflection: Navier-stokes simulations. Shock Waves, 511:363-379, 2004.

[37] G. S. Jiang and C. W. Shu. Efficient implementation of weighted ENO schemes. J. Comput. Physics, 126:202-228, 1996.

[38] P. Kaps and P. Rentrop. Generalized Runge-Kutta methods of order four with step size control for stiff ordinary differential equations. Num. Math., 33:55-68, 1979.

[39] R. J. Kee, F. M. Rupley, and J. A. Miller. Chemkin-II: A Fortran chemical kinetics package for the analysis of gas-phase chemical kinetics. Sand89-8009, Sandia National Laboratories, Livermore, California, 1989.

[40] C. A. Kennedy and M. H. Carpenter. Several new numerical methods for compressible shear layer simulations. Appl. Numer. Math., $14: 397-433,1994$.

[41] D. A. Kessler, V. N Gamezo, and E. S. Oran. Simulations of flame acceleration and deflagration-to-detonation transitions in methane-air systems. Combustion and Flame, 157:2063-2077, 2010.

[42] P. Knupp and S. Kambiz. Verification of Computer Codes in Computational Science and Engineering. Chapman and Hall/CRC, 2002.

[43] R. M. J. Kramer, C. Pantano, and D. I. Pullin. A class of energy stable, high-order finite-difference interface schemes suitable for adaptive mesh refinement of hyperbolic problems. J. Comput. Phys., 226:1458-1484, 2007.

[44] J. Larson and S. K. Lele. Direct numerical simulation of canonical shock/turbulence interaction. Phys. Fluids, $21,2009$.

[45] J. Larsson and B. Gustafsson. Stability criteria for hybrid difference methods. J. Comput. Physics, 227:2886-2898, 2008.

[46] S. K. Lele. Compact finite difference schemes with spectral like resolution. J. Comput. Phys., 103:16-42, 1992.

[47] R. J. Leveque. Finite Volume Methods for Hyperbolic Problems. Cambridge University Press, NY, 2002.

[48] Z. Liang, S. Browne, R. Deiterding, and Shepherd J. E. Detonation front structure and the competition for radicals. In Proc. Combust. Inst. 31, pages 2445-2453, 2007.

[49] M. Lombardini. Richtmyer-Meshkov instability in converging geometries. PhD thesis, California Institute of Technology, 2008.

[50] M. Lombardini and D. I. Pullin. Small-amplitude perturbations in the three-dimensional cylindrical Richtmyer-Meshkov instability. Physics of Fluids, 21, 2009.

[51] M. P. Martin, E. M. Taylor, M. Wu, and V. G. Weirs. A bandwidth-optimized WENO scheme for the effective direct numerical simulation of compressible turbulence. J. Comput. Physics, 220:270-289, 2006.

[52] L. Massa, J. M. Austin, and T. L. Jackson. Triple-point shear layers in gaseous detonation waves. J. Fluid Mech., 586:205-248, 2007.

[53] G. Matheou, A. M. Bonanos, C. Pantano, and P. E. Dimotakis. Large-eddy simulation of mixing in a recirculating shear flow. J. Fluid Mech., 646:375-414, 2010.

[54] S. Osher and S. Chakravarthy. High resolution schemes and the entropy condition. SIAM J. Numer. Anal., 21:955-984, 1984. 
[55] T. Pang. An Introduction to Computational Physics. Cambridge University Press, 1997.

[56] C. Pantano, R. Deiterding, D. J. Hill, and D. I. Pullin. A low numerical dissipation patch-based adaptive mesh refinement method for large-eddy simulation of compressible flows. J. Comput. Phys., 221(1):63-87, 2006.

[57] F. Pintgen, C. A. Eckett, J. M. Austin, J. E. Shepherd, and H. Hornung. Direct observations of reaction zone structure in propagating detonations. Combustion and Flame, 133(3):211-229, 2003

[58] S. Pirozzoli. Conservative hybrid compact-WENO schemes for shock-turbulence interaction. J. Comput. Physics, 178:81-117, 2002.

[59] A. Y. Poludnenko and E.S. Oran. The interaction of high-speed turbulence with flames: Global properties and internal flame structure. Combustion and Flame, 157:995-1011, 2010.

[60] M. I. Radulescu, G. J. Sharpe, C. K. Law, J. H. .S. Lee, C. B. Kiyanda, A. J. Higgens, and R. K. Hanson. The ignition mechanism in irregular structure gaseous detonations. In Proc. Combust. Inst. 30, pages 1859-1867, 2005.

[61] M. I. Radulescu, G. J. Sharpe, C. K. Law, and J. H. S. Lee. The hydrodynamic structure of unstable cellular detonations. J. Fluid Mech., 580:31-81, 2007.

[62] P. G. Saffman, M. J. Ablowitz, E. J. Hinch, J. R. Ockendon, and P. J. Olver. Vortex dynamics. Cambridge University Press, 2006.

[63] S. Sanderson, J. Austin, Z. Liang, F. Pintgen, J. E. Shepherd, and H. Hornung. Reactant jetting in unstable detonation. Progress in Aerospace Sciences, 46(2-3):116-131, 2010.

[64] J. E. Shepherd. Detonation in gases. In Proc. Combust. Inst. 32, pages 83-98, 2009.

[65] C. W. Shu. Essentially non-oscillatory and weighted essentially non-oscillatory schemes for hyperbolic conservation laws. Technical Report No. 97-65, Institute for Computer Applications in Science and Engineering NASA Langley Research Center, 1997.

[66] R. Wang and R. J. Spiteri. Linear instability of the fifth-order weno method. SIAM J. Numer. Anal., 45:1871-1901, 2007.

[67] G. Ward and D. I. Pullin. A hybrid, center-difference, limiter method for simulations of compressible multicomponent flows with Mie-Gruneisen equation of state. J. Comput. Physics, 229:2999-3018, 2010.

[68] V. G. Weirs and G. V. Candler. Optimization of weighted ENO schemes for DNS of compressible turbulence. AIAA, 97-1940:1-11, 1997.

[69] C. K. Westbrook. Chemical kinetics of hydrocarbon oxidation in gaseous detonations. Combustion $\& 3$ Flame, 46:191-210, 1982.

[70] F. A. Williams. Combustion theory. Addison-Wesley, Reading, MA, 1985. 